\title{
Three-Dimensional Vehicle-to-Vehicle Channel Modeling with Multiple Moving Scatterers
}

\author{
Derong Du, Xiaoping Zeng, Xin Jian, Lijuan Miao, and Haobo Wang \\ College of Communication Engineering, Chongqing University, Chongqing 400044, China \\ Correspondence should be addressed to Xin Jian; jianxin_zg@163.com
}

Received 30 November 2016; Revised 9 April 2017; Accepted 3 May 2017; Published 10 July 2017

Academic Editor: Barbara M. Masini

Copyright (C) 2017 Derong Du et al. This is an open access article distributed under the Creative Commons Attribution License, which permits unrestricted use, distribution, and reproduction in any medium, provided the original work is properly cited.

\begin{abstract}
Connected vehicles have received much attention in recent years due to their significant societal benefit and commercial value. However, a suitable channel model for vehicle-to-vehicle (V2V) communications is difficult to build due to the dynamic communication environment. In this paper, a three-dimensional (3D) geometrical propagation model that includes line-of-sight (LoS), single bounced (SB), and multiple bounced (MB) rays is proposed. Each of multiple scatterers in the model is moving with a random velocity in a random direction. Based on the geometrical propagation model, a generalized 3D reference model for narrowband multiple-input-multiple-output (MIMO) V2V multipath fading channels is developed. The corresponding spacetime correlation functions (ST-CFs), time correlation functions (T-CFs), and space correlation functions (S-CFs) are analytically investigated and numerically simulated in terms of various factors. Several notable ST-CFs for V2V and fixed-to-mobile (F2M) communications become the special cases of ST-CFs of the proposed model by adjusting the corresponding channel parameters. Finally, the theoretical results of the space-Doppler power spectral density (SD-PSD) are compared with the available measured data. The close agreements between the theoretical and measured SD-PSD curves confirm the utility and generality of the proposed model.
\end{abstract}

\section{Introduction}

Connected vehicles have the potential to improve the safety and efficiency of the automobile transportation [1], and they are expected to be a pillar of a smart society and to revolutionize the way people move. Different from the conventional fixed-to-mobile (F2M) cellular systems, vehicle-tovehicle $(\mathrm{V} 2 \mathrm{~V})$ is a kind of the mobile-to-mobile (M2M) communication which allows both the transmitter and receiver to be in motion [2]. Suitable channel models and channel characterizations are absolutely essential for successful design of V2V systems, where the quality of wireless links between vehicles can vary greatly and rapidly from one environment to another as one or both ends move [3].

Many M2M channel models have been proposed in various ways, some of which are summarized in [4], and these models have important reference values in modeling the V2V channel. The models in M2M communication environment can be traced back from two-dimensional (2D) [5-7] and three-dimensional (3D) [8-11] fixed scattering models to $2 \mathrm{D}$ [12-17] and 3D [18-20] moving scattering models. The M2M channel models with the assumption of stationary scatterers have been proposed in [5-11]. However, moving scatterers are unavoidable in M2M communications. Moving foliage, walking pedestrians, and passing vehicles are only a few examples of scatterers in motion, which can be observed in most of the real-world radio propagation environments $[14,21]$. The impact of moving scatterers on channel characteristics has been studied in [22-25] for 2D F2M cellular and fixed-to-fixed (F2F) communications with line-of-sight (LoS) and single bounced (SB) rays.

Recently, modeling of M2M/V2V channels in the presence of moving scatterers has been discussed in [12-20]. A nonstationary multiple-input-multiple-output (MIMO) V2V channel model based on the geometrical street model was derived, and the impact of fixed and moving clusters of scatterers on the channel statistics was studied in $[12,15]$. A single-input-single-output (SISO) V2V channel model was derived assuming a typical propagation scenario in which the local scatterers without specific constraints of positions 
moved with random velocities in random directions in [13, 14]. The impact of mobile and stationary scattering clusters on the Doppler spectrum was investigated for wideband V2V communication channels in an urban canyon oncoming environment in $[16,17]$. All previously reported models in [12-17] are 2D propagation models in which the rays are LoS, $\mathrm{SB}$, or double bounced (DB). However, this 2D assumption does not seem to be appropriate for many communication scenarios, for example, urban V2V communications in which the transmitter and receiver antenna arrays are often located in close proximity to or lower than the surrounding scatterers.

A 3D geometrical propagation model that included both stationary and moving scatterers around the transmitter and receiver was proposed in [18], and this work was extended to wideband channels in [19]. The models in [18, 19] took into account that the rays in $\mathrm{V} 2 \mathrm{~V}$ channels could be both $\mathrm{SB}$ and $\mathrm{DB}$; however, they imposed some constraints on the position of the local scatterers and assumed that the transmitter, receiver, and scatterers were in motion with constant velocities in $2 \mathrm{D}$ space. Therefore, the models in $[18,19]$ cannot fully capture the 3D spatial information. A preliminary investigation of the impact of multiple moving scatterers on the Doppler spectrum in 3D V2V communication scenarios was presented in [20]. However, the work of [20] did not investigate and take into account the space-time correlation functions (ST-CFs), scatterer velocity distributions, angle distributions, and so on.

It is unavoidable in $\mathrm{V} 2 \mathrm{~V}$ communications that the rays from the transmitter to receiver are multiple bounced (MB) by moving scatterers, especially in environments with highdensity scatterers, for example, urban area. However, to the best of the authors' knowledge, the model and statistical properties of $3 \mathrm{D} \mathrm{V} 2 \mathrm{~V}$ or $\mathrm{M} 2 \mathrm{M}$ channels in the presence of multiple moving scatterers have been investigated rarely so far. This paper strives to alleviate the current lack of analytical studies by investigating the model and statistical properties of a narrowband 3D MIMO V2V channel in which the local multiple scatterers are moving with random velocities in random directions. The proposed reference model constructs the channel impulse response as a combination of LoS and $\mathrm{MB}$ components, and SB is considered as a special case of MB. Different from the assumption of all non-LoS (NLoS) path gains having the same size in $[13,14]$ and meanwhile compared with models in $[18,19], \mathrm{SB}, \mathrm{DB}$, and other MB rays have more flexible and precise power weights in the proposed model. From the reference model, the corresponding STCFs, time correlation functions (T-CFs), and space correlation functions (S-CFs) are analytically investigated and numerically simulated in terms of various factors such as the maximum bounces, scattering forms, scatterer velocity distributions, and spacing between adjacent antenna elements. Finally, the theoretical space-Doppler power spectral density (SD-PSD) results with SD-PSDs in [18] and measured data in $[26,27]$ are compared. The close agreements between the analytically and empirically obtained SD-PSDs confirm the utility and generality of the proposed model and show the importance of including multiple moving scatterers in propagation models. The contributions and novelties of this paper are summarized as follows. (i) We propose a generalized geometrical model and a generalized reference model that include LoS, SB, and $\mathrm{MB}$ rays between the transmitter and receiver for 3D narrowband MIMO V2V communications. The proposed model can be adapted to a wide variety of scenarios, for example, F2F, F2M, and M2M with certain bounces by adjusting model parameters.

(ii) To the best of the authors' knowledge, the impact of rays' different maximum bounces caused by the multiple moving scatterers on the ST-CFs and T-CFs is deeply investigated for the first time in $\mathrm{V} 2 \mathrm{~V}$ or M2M communication environments.

(iii) The ST-CFs obtained from the proposed reference model are relatively generalized and can be reduced to several existing ST-CFs and T-CFs, for example, those in $[13,14,23,28,29]$.

The remainder of the paper is organized as follows. Section 2 describes the geometrical propagation model and presents a $3 \mathrm{D}$ reference model for narrowband MIMO V2V channels in the presence of multiple moving scatterers. Section 3 derives the channel ST-CFs, T-CFs, S-CFs, and SDPSDs for different parametric sets. Numerical results and comparison between the theoretical results and measured data are provided in Section 4. Finally, Section 5 provides some concluding remarks.

\section{3D Geometrical Propagation Model and Reference Model}

2.1. Geometrical Propagation Model. This paper considers the MIMO V2V communication links between transmitter $T_{X}$ and receiver $R_{X}$, as shown in Figure 1. The radio propagation environment is characterized by $3 \mathrm{D}$ multiple moving scattering with either LoS or NLoS conditions between $T_{X}$ and $R_{X}$. The MB waves emitted from the $q$ th antenna element of $T_{X}$ at an angle of departure (AOD) reach the $p$ th antenna element of $R_{X}$ at an angle of arrival (AOA) after being multiple scattered by the local moving scatterers.

For ease of reference, in this geometrical propagation model, the main parameters are summarized in Table 1, and the main assumptions are summarized as follows:

(i) The received signal power consists of LoS, SB, and MB scattering components with the corresponding power weights.

(ii) Both $T_{X}$ and $R_{X}$ are equipped with uniform linear arrays consisting of omnidirectional antenna elements.

(iii) $T_{X}$ and $R_{X}$ move with constant velocities in $3 \mathrm{D}$ space described by the fixed azimuth angles and elevation angles.

(iv) Each of the local scatterers on the links between $T_{X}$ and $R_{X}$ for the multiple bounces is in motion with a random velocity in a random direction in $3 \mathrm{D}$ space. 


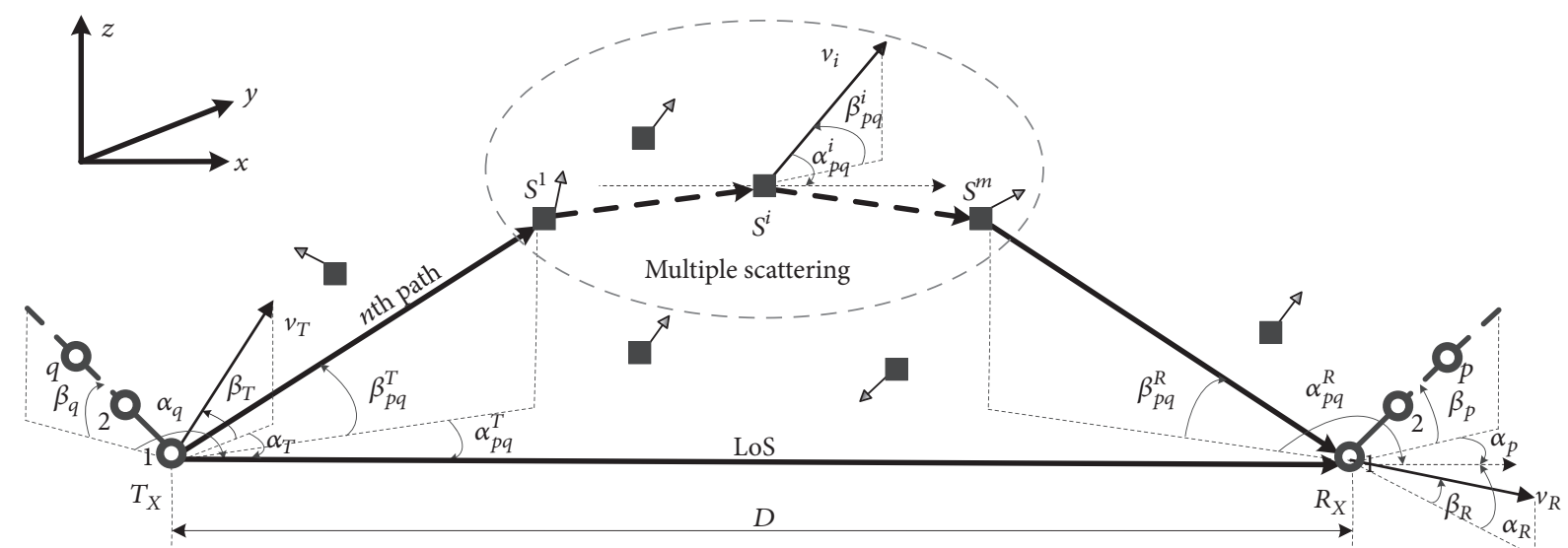

FIGURE 1: Geometrical propagation model with LoS and MB rays for the 3D MIMO V2V communication scenario. Empty circles represent the antenna elements, and solid squares represent the moving scatterers.

TABLE 1: Definition of the parameters used in the geometrical model.

\begin{tabular}{lcc}
\hline Parameters & Definition & Attributes \\
\hline$S^{i}$ & The $i$ th moving scatterer for the multiple bounces & Symbol \\
$D$ & The distance between $T_{X}$ and $R_{X}$ & Deterministic \\
$q, p$ & The antenna element identifier of $T_{X}$ and $R_{X}$, respectively & Deterministic \\
$Q, P$ & The number of antenna elements of $T_{X}$ and $R_{X}$, respectively & Deterministic \\
$d_{T}, d_{R}$ & The spacing between two adjacent antenna elements of $T_{X}$ and $R_{X}$, respectively & Deterministic \\
$\alpha_{q}, \beta_{q}$ & The azimuth angle and elevation angle of $T_{X}$ 's antenna array, respectively & Deterministic \\
$\alpha_{p}, \beta_{p}$ & The azimuth angle and elevation angle of $R_{X}$ 's antenna array, respectively & Deterministic \\
$\alpha_{p q}^{T}, \beta_{p q}^{T}$ & The azimuth angle and elevation angle of AOD, respectively & Statistical \\
$\alpha_{p q}^{R}, \beta_{p q}^{R}$ & The azimuth angle and elevation angle of AOA, respectively & Statistical \\
$v_{T}, v_{R}$ & The velocities of $T_{X}$ and $R_{X}$, respectively & Deterministic \\
$v_{p q}^{i}$ & The velocity of $S^{i}$ & Statistical \\
$\alpha_{T}, \beta_{T}$ & The azimuth angle and elevation angle of $v_{T}$, respectively & Deterministic \\
$\alpha_{R}, \beta_{R}$ & The azimuth angle and elevation angle of $v_{R}$, respectively & Deterministic \\
$\alpha_{p q}^{i}, \beta_{p q}^{i}$ & The azimuth angle and elevation angle of $v_{p q}^{i}$, respectively & Statistical \\
\hline
\end{tabular}

(v) The geometrical propagation model does not impose specific constraints on the position of the local moving scatterers like $[13,14]$. Owing to high path loss, we neglect the energy contribution of remote scatterers.

2.2. Reference Model. We can observe from Figure 1 that the complex faded envelope of the link between the $q$ th antenna element of $T_{X}$ and the $p$ th antenna element of $R_{X}$ can be written as a superposition of LoS and MB components; that is,

$$
\begin{aligned}
h_{p q}(t) & =h_{p q}^{\mathrm{LoS}}(t)+h_{p q}^{\mathrm{MB}}(t), \\
h_{p q}^{\mathrm{LoS}}(t) & =\rho_{p q} \\
\cdot \exp & \left\{j\left[2 \pi f_{p q, \rho} t+2 \pi\left(f_{p q, \rho}^{q}+f_{p q, \rho}^{p}\right)+\theta_{p q, \rho}\right]\right\}, \\
h_{p q}^{\mathrm{MB}}(t) & =\sum_{m=1}^{M} \sqrt{p_{m}} h_{p q}^{m}(t),
\end{aligned}
$$

$$
\begin{aligned}
& h_{p q}^{m}(t)=\lim _{N_{m} \rightarrow \infty} \sum_{n_{m}=1}^{N_{m}} c_{p q, n_{m}} \\
& \cdot \exp \left\{j\left[2 \pi f_{p q, n_{m}} t+2 \pi\left(f_{p q, n}^{q}+f_{p q, n}^{p}\right)+\theta_{p q, n_{m}}\right]\right\},
\end{aligned}
$$

where $\rho_{p q}, f_{p q, \rho}, \theta_{p q, \rho}, f_{p q, \rho}^{q}$, and $f_{p q, \rho}^{p}$ denote the path gain, Doppler shift, phase shift, frequency shift for $q$ th antenna element of $T_{X}$, and frequency shift for $p$ th antenna element of $R_{X}$ of $h_{p q}^{\mathrm{LoS}}(t)$, respectively; $m$ denotes the amount of bounces, $M$ is the maximum of $m, p_{m}$ and $N_{m}$ denote the power weight and path number of $h_{p q}^{m}(t)$, respectively; $c_{p q, n_{m}}, f_{p q, n_{m}}, \theta_{p q, n_{m}}$, $f_{p q, n}^{q}$, and $f_{p q, n}^{p}$ denote the path gain, Doppler shift, phase shift, frequency shift for $q$ th antenna element of $T_{X}$, and frequency shift for $p$ th antenna element of $R_{X}$ of the $n_{m}$ th path in $h_{p q}^{m}(t)$. The LoS component $h_{p q}^{\mathrm{LoS}}(t)$ can be described by a complex sinusoid, and (2) is an extension of the (3.17) in [30]. $h_{p q}^{m}(t)$ denotes the NLoS component with $m$ bounced 
$(m \mathrm{~B})$ rays, and it is an extension of the (3.12) in [30]. Note that $\mathrm{MB}$ component $h_{p q}^{\mathrm{MB}}(t)$ of the channel impulse response consists of $M$ clusters of rays reflected $m \in\{1,2,3, \ldots, M\}$ times from moving scatterers.

The channel gains $\left(\rho_{p q}, c_{p q, n_{m}}\right)$, Doppler shifts and frequency shifts $\left(f_{p q, \rho}, f_{p q, \rho}^{q}, f_{p q, \rho}^{p}, f_{p q, n_{m}}, f_{p q, n}^{q}, f_{p q, n}^{p}\right)$, and phases $\left(\theta_{p q, \rho}, \theta_{p q, n_{m}}\right)$ in this model can be calculated as follows.

2.2.1. Channel Gains. The central limit theorem states that $h_{p q}^{m}(t)$ equals a complex valued Gaussian random process with zero mean and variance $2 \sigma_{m}^{2}=\operatorname{Var}\left\{h_{p q}^{m}(t)\right\}=$ $\lim _{N_{m} \rightarrow \infty} \sum_{n_{m}}^{N_{m}} E\left[c_{p q, n_{m}}^{2}\right] . p_{m}$ in (3) denotes the power weight of the $m$ th clusters of rays, and $\sum_{m=1}^{M} p_{m}=1$. The channel gain of $h_{p q}(t)$ is normalized, i.e., $2 \sum_{m=1}^{M} p_{m} \sigma_{m}^{2}+\rho_{p q}^{2}=1$, and the Rice factor can be denoted as $K=\rho_{p q}^{2} / 2 \sum_{m=1}^{M} p_{m} \sigma_{m}^{2}$. These parameters have to be either set during simulations or estimated from measurements.

2.2.2. Doppler Shifts and Frequency Shifts. The frequency shifts $f_{p q, \rho}^{q}, f_{p q, \rho}^{p}, f_{p q, n}^{q}$, and $f_{p q, n}^{p}$ depend on the difference of the propagation distance (TPD) changes between $h_{p q}(t)$ and $h_{11}(t)$. On the other hand, the Doppler shifts $f_{p q, \rho}$ and $f_{p q, n_{m}}$ depend on the geometrical relation between directions of movement of $T_{X}, R_{X}$, and multiple moving scatterers and the directions of AOD and AOA. For $\max \left(d_{T}, d_{R}\right) \ll D$, both the AOD and AOA of LoS rays are approximately equal to zero. Appendix A shows that $f_{p q, \rho}^{q}, f_{p q, \rho}^{p}, f_{p q, n}^{q}, f_{p q, n}^{p}$, and $f_{p q, \rho}$ are, respectively,

$$
\begin{aligned}
f_{p q, \rho}^{q} & =\frac{(q-1) d_{T} f_{0}}{c} \cos \alpha_{q} \cos \beta_{q}, \\
f_{p q, \rho}^{p} & =\frac{(p-1) d_{R} f_{0}}{c} \cos \alpha_{p} \cos \beta_{p}, \\
f_{p q, n}^{q} & =\frac{(q-1) d_{T} f_{0}}{c}\left[\cos \left(\alpha_{q}-\alpha_{p q}^{T}\right) \cos \beta_{q} \cos \beta_{p q}^{T}\right. \\
+ & \left.\sin \beta_{q} \sin \beta_{p q}^{T}\right], \\
f_{p q, n}^{p} & =\frac{(p-1) d_{R} f_{0}}{c}\left[\cos \left(\alpha_{p}-\alpha_{p q}^{R}\right) \cos \beta_{p} \cos \beta_{p q}^{R}\right. \\
+ & \left.\sin \beta_{p} \sin \beta_{p q}^{R}\right], \\
f_{p q, \rho} & =\frac{f_{0}}{c}\left(v_{T} \cos \alpha_{T} \cos \beta_{T}+v_{R} \cos \alpha_{R} \cos \beta_{R}\right),
\end{aligned}
$$

where $f_{0}$ is the carrier frequency and $c$ denotes the speed of light.

$$
f_{p q, n_{m}}=f_{p q}^{T}+f_{p q}^{R}+f_{p q, n_{m}}^{\mathrm{AOD}}+f_{p q, n_{m}}^{\mathrm{AOA}}
$$

where $f_{p q}^{T}$ and $f_{p q}^{R}$ are caused by the movement of $T_{X}$ and $R_{X}$, respectively. $f_{p q, n_{m}}^{\mathrm{AOD}}$ and $f_{p q, n_{m}}^{\mathrm{AOA}}$ are caused by the movement of $m$ scatterers relative to the directions of AOD and AOA, respectively. Using the similar mathematical manipulations in Appendix A, the respective components in (10) are

$$
\begin{aligned}
f_{p q}^{T} & =\frac{v_{T} f_{0}}{c}\left[\cos \left(\alpha_{T}-\alpha_{p q}^{T}\right) \cos \beta_{T} \cos \beta_{p q}^{T}\right. \\
& \left.+\sin \beta_{T} \sin \beta_{p q}^{T}\right], \\
f_{p q}^{R} & =\frac{v_{R} f_{0}}{c}\left[\cos \left(\alpha_{R}-\alpha_{p q}^{R}\right) \cos \beta_{R} \cos \beta_{p q}^{R}\right. \\
& \left.+\sin \beta_{R} \sin \beta_{p q}^{R}\right] .
\end{aligned}
$$

According to [31], the maximum Doppler shift for MB link over $m$ moving scatterers with velocities $v_{i} \ll c$ is

$$
f_{m}^{\max }=\frac{f_{0}}{c}\left(v_{1}+2 \sum_{i=2}^{m-1} v_{i}+v_{m}\right) .
$$

Therefore, the remainder components of $f_{p q, n_{m}}$ are [20]

$$
\begin{aligned}
& f_{p q, n_{m}}^{\mathrm{AOD}} \\
& \approx \frac{f_{0}}{c}\left(v_{p q}^{1} P_{p q}^{\mathrm{AOD}, 1}+2 \sum_{i=2}^{m-1} v_{p q}^{i} P_{p q}^{\mathrm{AOD}, i}+v_{p q}^{m} P_{p q}^{\mathrm{AOD}, m}\right) \\
& f_{p q, n_{m}}^{\mathrm{AOA}} \\
& \approx \frac{f_{0}}{c}\left(v_{p q}^{1} P_{p q}^{\mathrm{AOA}, 1}+2 \sum_{i=2}^{m-1} v_{p q}^{i} P_{p q}^{\mathrm{AOA}, i}+v_{p q}^{m} P_{p q}^{\mathrm{AOA}, m}\right), \\
& P_{p q}^{\mathrm{AOD}, i}=\cos \left(\alpha_{p q}^{i}-\alpha_{p q}^{T}\right) \cos \beta_{p q}^{i} \cos \beta_{p q}^{T} \\
& +\sin \beta_{p q}^{i} \sin \beta_{p q}^{T} \text {, } \\
& P_{p q}^{\mathrm{AOA}, i}=\cos \left(\alpha_{p q}^{i}-\alpha_{p q}^{R}\right) \cos \beta_{p q}^{i} \cos \beta_{p q}^{R} \\
& +\sin \beta_{p q}^{i} \sin \beta_{p q}^{R} \text {. }
\end{aligned}
$$

Note that if $\beta_{T}=\beta_{R}=\beta_{p q}^{T}=\beta_{p q}^{R}=\beta_{p q}^{i}=0$ and $m=1$, (10) equals (7) in [13] and (5) in [14] regardless of the plus or minus signs. The differences among these plus or minus signs are caused by the different forms of angles' expression.

2.2.3. Phases. The phase shift $\theta_{p q, \rho}$ can be assumed to be constant [30]. The phase shift $\theta_{p q, n_{m}}$ consists of the phase change caused by the interaction of the transmitted signal with the scatterers and the phase change caused by the TPD between the first and the last scatterers. Without loss of generality, we can assume that the phases $\theta_{p q, n_{m}}(m=$ $1,2,3, \ldots, M)$ are independent random variables. Here, it is assumed that they are uniformly distributed on the interval $[0,2 \pi)$ and independent of any other random variable. 


\section{Space-Time Correlation Function and Space-Doppler Power Spectral Density}

Using the reference model described in Section 2, we can now derive the key temporal and spatial characteristics of MIMO V2V narrowband multipath fading channels with the local multiple moving scatters.

3.1. Space-Time Correlation Function. The normalized ST-CF between two complex faded envelopes $h_{p q}(t)$ and $h_{\bar{p} \bar{q}}(t)$ is defined as

$$
R_{\bar{p} \bar{q}, p q}\left(d_{T}, d_{R}, \tau\right)=E\left[h_{\bar{p} \bar{q}}(t)^{*} h_{p q}(t+\tau)\right],
$$

where $(\bullet)^{*}$ denotes the complex conjugate operation, $E(\bullet)$ is the statistical expectation operator, $p, \bar{p} \in\{1,2,3, \ldots, P\}$, and $q, \bar{q} \in\{1,2,3, \ldots, Q\}$. The normalized T-CF can be obtained, if $p=\bar{p}$ and $q=\bar{q}$ in (15). The normalized space correlation function (S-CF) can be obtained by setting $\tau$ to zero in (15).

Since $h_{p q}^{1}(t), h_{p q}^{2}(t), \ldots, h_{p q}^{M}(t)$, and $h_{p q}^{\text {LoS }}(t)$ are independent of each other, (15) can be simplified to

$$
\begin{aligned}
R_{\bar{p} \bar{q}, p q}\left(d_{T}, d_{R}, \tau\right)= & R_{\bar{p} \bar{q}, p q}^{\mathrm{MB}}\left(d_{T}, d_{R}, \tau\right) \\
& +R_{\bar{p} \bar{q}, p q}^{\mathrm{LoS}}\left(d_{T}, d_{R}, \tau\right) \\
= & \sum_{m=1}^{M} p_{m} R_{\bar{p} \bar{q}, p q}^{m}\left(d_{T}, d_{R}, \tau\right) \\
& +R_{\bar{p} \bar{q}, p q}^{\operatorname{Los}}\left(d_{T}, d_{R}, \tau\right),
\end{aligned}
$$

where $R_{\bar{p} \bar{q}, p q}^{m}\left(d_{T}, d_{R}, \tau\right)$ and $R_{\bar{p} \bar{q}, p q}^{\operatorname{LoS}}\left(d_{T}, d_{R}, \tau\right)$ denote the normalized ST-CFs of the $m \mathrm{~B}$ and LoS components, respectively, and they are defined as

$$
\begin{aligned}
& R_{\bar{p} \bar{q}, p q}^{m}\left(d_{T}, d_{R}, \tau\right)=E\left[h_{\bar{p} \bar{q}}^{m}(t)^{*} h_{p q}^{m}(t+\tau)\right], \\
& R_{\bar{p} \bar{q}, p q}^{\operatorname{LoS}}\left(d_{T}, d_{R}, \tau\right)=E\left[h_{\bar{p} \bar{q}}^{\operatorname{LoS}}(t)^{*} h_{p q}^{\operatorname{LoS}}(t+\tau)\right] .
\end{aligned}
$$

$$
\begin{aligned}
& R_{\bar{p} \bar{q}, p q}^{m}\left(d_{T}, d_{R}, \tau\right) \\
& =\lim _{N_{n} \rightarrow \infty} \lim _{N_{m} \rightarrow \infty} \sum_{n_{n}=1}^{N_{n}} c_{\bar{p} \bar{q}, n_{n}} \sum_{n_{m}=1}^{N_{m}} c_{p q, n_{m}} \\
& \quad \times E\left\{\exp \left\{j\left[2 \pi\left(f_{p q, n_{m}}-f_{\bar{p} \bar{q}, n_{n}}\right) t+2 \pi f_{p q, n_{m}} \tau+2 \pi\left(f_{p q, n}^{q}+f_{p q, n}^{p}-f_{\bar{p} \bar{q}, n}^{\bar{q}}-f_{\bar{p} \bar{q}, n}^{\bar{p}}\right)+\theta_{p q, n_{m}}-\theta_{\bar{p} \bar{q}, n_{n}}\right]\right\}\right\} .
\end{aligned}
$$

Under the assumption that $\theta_{p q, n_{m}}$ and $\theta_{\bar{p} \bar{q}, n_{n}}$ are uniformly distributed on the interval $[0,2 \pi)$ and independent of each other, $E\left\{\exp \left[j\left(\theta_{p q, n_{m}}-\theta_{\bar{p} \bar{q}, n_{n}}\right)\right]\right\}$ equals 1 . It is assumed that all the path gains of the $m \mathrm{~B}$ component have the same size; that is,

$$
c_{\bar{p} \bar{q}, n_{n}}=c_{p q, n_{m}}=\sigma_{m} \sqrt{\frac{2}{N_{m}}} .
$$

3.1.1. ST-CF of LoS Component. By substituting (2) into (18), the expression for the ST-CF of the LoS component can be written as

$$
\begin{aligned}
& R_{\bar{p} \bar{q}, p q}^{\mathrm{LoS}}\left(d_{T}, d_{R}, \tau\right) \\
& \quad=\rho_{p q} \rho_{\bar{p} \bar{q}} E\left\{\operatorname { e x p } \left\{j \left[2 \pi t\left(f_{p q, \rho}-f_{\bar{p} \bar{q}, \rho}\right)+2 \pi f_{p q, \rho} \tau\right.\right.\right. \\
& \quad+2 \pi\left(f_{p q, \rho}^{q}+f_{p q, \rho}^{p}-f_{\bar{p} \bar{q}, \rho}^{\bar{q}}-f_{\bar{p} \bar{q}, \rho}^{\bar{p}}\right)+\theta_{p q, \rho} \\
& \left.\left.\left.\quad-\theta_{\bar{p} \bar{q}, \rho}\right]\right\}\right\} .
\end{aligned}
$$

For $\max \left(d_{T}, d_{R}\right) \ll D$, we assume $\rho_{p q}=\rho_{\bar{p} \bar{q}}$ and $\theta_{p q, \rho}=$ $\theta_{\bar{p} \bar{q}, \rho}$. Then, (19) can be written as

$$
\begin{aligned}
R_{\bar{p} \bar{q}, p q}^{\operatorname{LoS}}\left(d_{T}, d_{R}, \tau\right) & =\rho_{p q}^{2} E\left\{\exp \left[j 2 \pi\left(f_{p q, \rho} \tau+\Delta f_{\rho}\right)\right]\right\} \\
& =\rho_{p q}^{2} \exp \left[j 2 \pi\left(f_{p q, \rho} \tau+\Delta f_{\rho}\right)\right],
\end{aligned}
$$

where $\Delta f_{\rho}$ is defined as

$$
\Delta f_{\rho}=f_{p q, \rho}^{q}+f_{p q, \rho}^{p}-f_{\bar{p} \bar{q}, \rho}^{\bar{q}}-f_{\bar{p} \bar{q}, \rho}^{\bar{p}} .
$$

By substituting (5) and (6) into (21), $\Delta f_{\rho}$ can be written as

$$
\begin{gathered}
\Delta f_{\rho}=\frac{f_{0}}{c}\left[(q-\bar{q}) d_{T} \cos \alpha_{q} \cos \beta_{q}\right. \\
\left.+(p-\bar{p}) d_{R} \cos \alpha_{p} \cos \beta_{p}\right] .
\end{gathered}
$$

3.1.2. ST-CF of $m B$ Component. By substituting (4) into (17), the expression for the ST-CF of the $m \mathrm{~B}$ component can be written as
Then, (23) can be written as

$$
\begin{aligned}
& R_{\bar{p} \bar{q}, p q}^{m}\left(d_{T}, d_{R}, \tau\right) \\
& \quad=2 \sigma_{m_{N_{m} \rightarrow \infty}^{2}} \lim _{n_{m}=1} \sum_{m}^{N_{m}} E\left\{\exp \left[j 2 \pi\left(f_{p q, n_{m}} \tau+\Delta f_{n_{m}}\right)\right]\right\},
\end{aligned}
$$


where $\Delta f_{n_{m}}$ is defined as

$$
\Delta f_{n_{m}}=f_{p q, n}^{q}+f_{p q, n}^{p}-f_{\bar{p} \bar{q}, n}^{\bar{q}}-f_{\bar{p} \bar{q}, n}^{\bar{p}}
$$

It is assumed that AOD and AOA of $h_{p q}^{m}(t)$ and $h_{\bar{p} \bar{q}}^{m}(t)$ are independent and identically distributed (i.i.d.). By substituting (7) and (8) into (26), $\Delta f_{n_{m}}$ can be written as

$$
\begin{aligned}
& \Delta f_{n_{m}}=\frac{f_{0}}{c}\{(q \\
& -\bar{q}) d_{T}\left[\cos \left(\alpha_{q}-\alpha_{p q}^{T}\right) \cos \beta_{q} \cos \beta_{p q}^{T}+\sin \beta_{q} \sin \beta_{p q}^{T}\right]
\end{aligned}
$$

$$
+(p
$$$$
\left.-\bar{p}) d_{R}\left[\cos \left(\alpha_{p}-\alpha_{p q}^{R}\right) \cos \beta_{p} \cos \beta_{p q}^{R}+\sin \beta_{p} \sin \beta_{p q}^{R}\right]\right\} .
$$

Since the number of local scatterers in the reference model is infinite, the parameters $\alpha_{p q}^{T}, \beta_{p q}^{T}, \alpha_{p q}^{R}, \beta_{p q}^{R}, \alpha_{p q}^{i}$, and $\beta_{p q}^{i}$ can be seen as continuous random variables with corresponding probability density functions (PDFs). Then, the ST-CF of the $m \mathrm{~B}$ component (25) can be written as

$$
\begin{aligned}
& R_{\overline{p \bar{q}, p q}}^{m}\left(d_{T}, d_{R}, \tau\right)=2 \sigma_{m}^{2} \\
& \quad \times \int \cdots \iint \cdots \iint \cdots \iiint \iint \exp \left[j 2 \pi\left(f_{p q, n_{m}} \tau+\Delta f_{n_{m}}\right)\right] \\
& \quad \times \prod_{i=1}^{m} p\left(\nu_{p q}^{i}\right) \prod_{i=1}^{m} p\left(\alpha_{p q}^{i}, \beta_{p q}^{i}\right) \\
& \quad \cdot p\left(\alpha_{p q}^{T}, \beta_{p q}^{T}, \alpha_{p q}^{R}, \beta_{p q}^{R}\right) d \nu_{p q}^{1} \cdots d v_{p q}^{m} d \alpha_{p q}^{1} \cdots d \alpha_{p q}^{m} d \beta_{p q}^{1} \cdots d \beta_{p q}^{m} d \alpha_{p q}^{T} d \beta_{p q}^{T} d \alpha_{p q}^{R} d \beta_{p q}^{R} .
\end{aligned}
$$

Now, the complete expression of (16) can be obtained by substituting (20) and (28) into (16). $p\left(\alpha_{p q}^{T}, \beta_{p q}^{T}, \alpha_{p q}^{R}, \beta_{p q}^{R}\right)$ describes the joint distribution of AOD and AOA, and it can be optionally used to present some propagation channel models. As a result, the ST-CF in (28) can provide a suitable platform to study the statistical properties of some different channel models, such as the random scattering model $[13,14]$, Jakes model [28], one-ring model [23], and two-ring model [29] as described in Section 3.1.3. Therefore, the ST-CF in (28) is a generalized and parametric expression. However, due to the complex nature of $p\left(\alpha_{p q}^{T}, \beta_{p q}^{T}, \alpha_{p q}^{R}, \beta_{p q}^{R}\right)$, it is assumed that AOD and AOA are independent $[13,14,32,33]$, and azimuth angles and elevation angles in (28) are also independent $[18,19,32]$. The parameters in (28) such as the velocities of the multiple moving scatterers and random angles can be calculated as follows.

(i) Scatterer Velocity Distributions. The Gaussian, Laplace, exponential, and uniform distributions can be used to describe the velocity of moving scatterers [13]. In fact, the scatterer velocity $v_{p q}^{i}$ is always positive or equal to zero. We use the uniform distribution in (29) and half-Gaussian distribution in (30) to describe the velocity of multiple moving scatterers.

$$
p\left(v_{p q}^{i}\right)=\frac{1}{v_{\max }^{i}}, \quad 0 \leq v_{p q}^{i} \leq v_{\max }^{i}
$$

where $v_{\max }^{i}$ is the maximum of $v_{p q}^{i}$.

$$
p\left(v_{p q}^{i}\right)=\frac{\sqrt{2}}{\sqrt{\pi} \sigma_{p q, i}} \exp \left[-\frac{v_{p q}^{i}{ }^{2}}{2 \sigma_{p q, i}^{2}}\right], \quad v_{p q}^{i} \geq 0,
$$

where $\sigma_{p q, i}$ is the standard deviation of $\nu_{p q}^{i}$.

(ii) Angle Distributions. To characterize the statistical angles in Table 1, we use the uniform distribution in (31) in the isotropic scattering environment and use the von Mises distribution in (32) and the cosine distribution in (33) in the nonisotropic scattering environment. In addition, the interval of azimuth angles $\alpha_{p q}^{T}, \alpha_{p q}^{R}$, and $\alpha_{p q}^{i}$ is $(-\pi, \pi]$, and the interval of elevation angles $\beta_{p q}^{T}, \beta_{p q}^{R}$, and $\beta_{p q}^{i}$ is $(-\pi / 2, \pi / 2]$.

$$
\begin{aligned}
& p(\gamma)=\frac{1}{\gamma_{2}-\gamma_{1}}, \quad \gamma_{1} \leq \gamma \leq \gamma_{2}, \\
& p(\alpha)=\frac{\exp [k \cos (\alpha-\bar{\alpha})]}{2 \pi I_{0}(k)}, \quad \alpha_{1} \leq \alpha \leq \alpha_{1}+2 \pi,
\end{aligned}
$$

where $I_{0}(\bullet)$ is the zeroth-order modified Bessel function of the first kind, $\bar{\alpha}$ is the mean angle, and $k$ controls the spread of angles around the mean. The von Mises distribution PDF with $\bar{\alpha}=0$ is used to describe the azimuth angles.

$$
p(\beta)=\frac{\pi}{4\left|\beta_{m}\right|} \cos \left(\frac{\pi}{2} \frac{\beta}{\beta_{m}}\right), \quad-\beta_{m} \leq \beta \leq \beta_{m},
$$

where $\beta_{m}$ is the maximum of $\beta$. The cosine distribution PDF is used to describe the elevation angles.

3.1.3. Special Cases of $m B$ 's ST-CF. If $\beta_{T}=\beta_{R}=\beta_{p q}^{T}=$ $\beta_{p q}^{R}=\beta_{p q}^{i}=0$, that is, the scattering environment is $2 \mathrm{D}$, 
some different special cases can be derived from the general expression of the $m$ B's ST-CF in (28).

In the $2 \mathrm{D}$ scattering environment, (28) can be written as (B.2) in Appendix B. If $p=\bar{p}, q=\bar{q}$, and $m=1$, (B.2) equals (11) in [13] and (6) in [14] regardless of the plus or minus signs under the assumption that the angles $\alpha^{T}$ and $\alpha^{R}$ are independent of each other in $[13,14]$. In isotropic scattering environments, some other special cases with closed-form expressions can be derived as follows.

Appendix B shows that an approximate ST-CF of (B.2) can be written as

$$
\begin{aligned}
& R_{\bar{p} \bar{q}, p q}^{m}\left(d_{T}, d_{R}, \tau\right)=2 \sigma_{m}^{2} J_{0}\left\{k_{0}\left[v_{T} \tau+(q-\bar{q}) d_{T}\right]\right\} \\
& \quad \times J_{0}\left\{k_{0}\left[v_{R} \tau+(p-\bar{p}) d_{R}\right]\right\} \int \cdots \int J_{0}\left(2 k_{0} v_{p q}^{1} \tau\right) \\
& \quad \times J_{0}\left(2 k_{0} v_{p q}^{m} \tau\right) \prod_{i=2}^{m-1} J_{0}\left(4 k_{0} v_{p q}^{i} \tau\right) \\
& \quad \cdot \prod_{i=1}^{m} p\left(v_{p q}^{i}\right) d v_{p q}^{1} \cdots d v_{p q}^{m},
\end{aligned}
$$

where $J_{0}(\bullet)$ denotes the zeroth-order Bessel function of the first kind and $k_{0}=2 \pi f_{0} / c$ is the wave number.

Note that if $m$ approaches to the infinity, (34) can be written as

$$
\begin{aligned}
& \lim _{m \rightarrow \infty} R_{\bar{p} \bar{q}, p q}^{m}\left(d_{T}, d_{R}, \tau\right) \\
& = \begin{cases}2 \sigma_{m}^{2} J_{0}\left[(q-\bar{q}) k_{0} d_{T}\right] J_{0}\left[(p-\bar{p}) k_{0} d_{R}\right], & \tau=0, \\
0 & \tau>0 .\end{cases}
\end{aligned}
$$

In the NLoS communication environment with very high-density scatterers, (35) implies that the ST-CF approaches to zero at the nonzero time difference $\tau$ and has nothing to do with velocities of scatterers, $T_{X}$ and $R_{X}$. The large-scale antenna arrays are very suitable to be used in this environment because the antenna element spacing can be reduced to a smaller value.

If $v_{p q}^{i}$ is constant, (34) can be presented as the following closed-form expression:

$$
\begin{aligned}
R_{\bar{p} \bar{q}, p q}^{m} & \left(d_{T}, d_{R}, \tau\right) \\
= & 2 \sigma_{m}^{2} J_{0}\left\{k_{0}\left[v_{T} \tau+(q-\bar{q}) d_{T}\right]\right\} \\
& \times J_{0}\left\{k_{0}\left[v_{R} \tau+(p-\bar{p}) d_{R}\right]\right\} J_{0}\left(2 k_{0} v_{p q}^{1} \tau\right) \\
& \times J_{0}\left(2 k_{0} v_{p q}^{m} \tau\right) \prod_{i=2}^{m-1} J_{0}\left(4 k_{0} v_{p q}^{i} \tau\right) .
\end{aligned}
$$

The T-CF of the classical F2M scenario with fixed scatterers is obtained, if $v_{T}=v_{p q}^{i}=0(i=1,2,3, \ldots, m)$, $q=\bar{q}$, and $p=\bar{p}$ in (36). In this case, the ST-CF in (36) is $2 \sigma_{m}^{2} J_{0}\left(k_{0} v_{R} \tau\right)$, which is known as the Jakes model [28]. If $v_{T}=0, m=1, q=\bar{q}$, and $p=\bar{p}$, the ST-CF in (36) results in $2 \sigma_{m}^{2} J_{0}\left(k_{0} v_{p q}^{1} \tau\right) J_{0}\left(k_{0} v_{R} \tau\right)$, which equals the
T-CF of F2M single-ring channel model in the presence of moving scatterers reported in (11) of [23]. If $v_{p q}^{i}=0(i=$ $1,2,3, \ldots, m), q=\bar{q}$, and $p=\bar{p}$, the ST-CF in (36) results in $2 \sigma_{m}^{2} J_{0}\left(k_{0} v_{T} \tau\right) J_{0}\left(k_{0} v_{R} \tau\right)$, which equals the T-CFs of the classical M2M two-ring channel model in the presence of fixed scatterers reported in (46) of [29]. If $v_{p q}^{i}=\tau=0(i=$ $1,2,3, \ldots, m), q=p=2$, and $\bar{q}=\bar{p}=1$, the ST-CF in (36) results in $2 \sigma_{m}^{2} J_{0}\left(k_{0} d_{T}\right) J_{0}\left(k_{0} d_{R}\right)$, which equals the S-CF in (46) of [29].

The velocities of moving scatterers such as moving foliage, walking pedestrians, and passing vehicles generally are random variables. If $v_{p q}^{i}$ is described by the uniform distribution in (29), (34) can be presented as the following closed-form expression:

$$
\begin{aligned}
& R_{\overline{p \bar{q}, p q}}^{m}\left(d_{T}, d_{R}, \tau\right)=\frac{\sigma_{m}^{2}}{2^{m-1}} J_{0}\left\{k_{0}\left[v_{T} \tau+(q-\bar{q}) d_{T}\right]\right\} \\
& \quad \times J_{0}\left\{k_{0}\left[v_{R} \tau+(p-\bar{p}) d_{R}\right]\right\}\left[2 J_{0}\left(2 k_{0} v_{\max }^{1} \tau\right)\right. \\
& +\pi J_{1}\left(2 k_{0} v_{\max }^{1} \tau\right) H_{0}\left(2 k_{0} v_{\max }^{1} \tau\right)-\pi J_{0}\left(2 k_{0} v_{\max }^{1} \tau\right) \\
& \left.\quad \times H_{1}\left(2 k_{0} v_{\max }^{1} \tau\right)\right]\left[2 J_{0}\left(2 k_{0} v_{\max }^{m} \tau\right)\right. \\
& +\pi J_{1}\left(2 k_{0} v_{\max }^{m} \tau\right) H_{0}\left(2 k_{0} v_{\max }^{m} \tau\right)-\pi J_{0}\left(2 k_{0} v_{\max }^{m} \tau\right) \\
& \left.\quad \cdot H_{1}\left(2 k_{0} v_{\max }^{m} \tau\right)\right] \times \prod_{i=2}^{m-1}\left[2 J_{0}\left(4 k_{0} v_{\max }^{i} \tau\right)\right. \\
& \quad+\pi J_{1}\left(4 k_{0} v_{\max }^{i} \tau\right) \times H_{0}\left(4 k_{0} v_{\max }^{i} \tau\right) \\
& \left.\quad-\pi J_{0}\left(4 k_{0} v_{\max }^{i} \tau\right) H_{1}\left(4 k_{0} v_{\max }^{i} \tau\right)\right]
\end{aligned}
$$

where $J_{1}(\bullet)$ denotes the first-order Bessel function of the first kind, $H_{0}(\bullet)$ denotes the zeroth-order Struve function, and $H_{1}(\bullet)$ denotes the first-order Struve function.

If $v_{p q}^{i}$ is described by the half-Gaussian distribution in (30), (34) can be presented as the following closed-form expression:

$$
\begin{aligned}
& R_{\bar{p} \bar{q}, p q}^{m}\left(d_{T}, d_{R}, \tau\right)=2 \sigma_{m}^{2} J_{0}\left\{k_{0}\left[v_{T} \tau+(q-\bar{q}) d_{T}\right]\right\} \\
& \quad \times J_{0}\left\{k_{0}\left[v_{R} \tau+(p-\bar{p}) d_{R}\right]\right\} I_{0}\left(k_{0}^{2} \sigma_{p q, 1}^{2} \tau^{2}\right) \\
& \quad \times I_{0}\left(k_{0}^{2} \sigma_{p q, m}^{2} \tau^{2}\right) \exp \left(-k_{0}^{2} \sigma_{p q, 1}^{2} \tau^{2}\right) \\
& \quad \cdot \exp \left(-k_{0}^{2} \sigma_{p q, m}^{2} \tau^{2}\right) \\
& \quad \times \prod_{i=2}^{m-1} I_{0}\left(4 k_{0}^{2} \sigma_{p q, i}^{2} \tau^{2}\right) \exp \left(-4 k_{0}^{2} \sigma_{p q, i}^{2} \tau^{2}\right) .
\end{aligned}
$$

3.2. Space-Doppler Power Spectral Density. The SD-PSD can be obtained by taking the Fourier transform of the ST-CF in (16) with respect to time difference $\tau$. From (16), it follows that 
TABLE 2: Parameters used in the numerical simulation.

\begin{tabular}{lccccc}
\hline Parameters & Figures 2,3 , and 6 & Figures 4,5 , and 7 & Figures 8 and 9 & Figure 10 & Figure 11 \\
\hline$f_{0}(\mathrm{GHz})$ & 2.435 & 2.435 & 2.435 & 2.435 \\
$K$ & 2 & 2 & 2 & 2.5 & -41 \\
$k$ & 5.4 & 5.4 & 3 & 3 \\
$q, p$ & 2,2 & 1,1 & 2,2 & 1,1 & 1,1 \\
$\bar{q}, \bar{p}$ & 1,1 & 1,1 & variable & 0,0 & 1,1 \\
$d_{T}, d_{R}(\lambda)$ & $1 / 2,1 / 2$ & 0,0 & $\pi / 2, \pi / 6$ & $\pi / 2, \pi / 6$ & 0,0 \\
$\alpha_{q}, \beta_{q}(\mathrm{rad})$ & $\pi / 2, \pi / 6$ & $\pi / 2, \pi / 6$ & $\pi / 2, \pi / 6$ & 0,0 \\
$\alpha_{p}, \beta_{p}(\mathrm{rad})$ & $\pi / 2, \pi / 6$ & $\pi / 2, \pi / 6$ & 25,25 & 0,0 \\
$v_{T}, v_{R}(\mathrm{~m} / \mathrm{s})$ & 25,25 & 25,25 & $\pi / 2,0$ & 0,0 \\
$\alpha_{T}, \beta_{T}(\mathrm{rad})$ & $\pi / 2,0$ & $\pi / 2,0$ & $\pi / 2,0$ & 0,0 \\
$\alpha_{R}, \beta_{R}(\mathrm{rad})$ & $\pi / 2,0$ & $\pi / 2,0$ & & $\pi / 2,0$ \\
\hline
\end{tabular}

the SD-PSD is a summation of the SD-PSDs of the $M B$ and LoS components; that is,

$$
\begin{aligned}
S_{\bar{p} \bar{q}, p q}\left(d_{T}, d_{R}, \omega\right)= & \mathscr{F}_{\tau}\left\{R_{\bar{p} \bar{q}, p q}\left(d_{T}, d_{R}, \tau\right)\right\} \\
= & \sum_{m=1}^{M} p_{m} S_{\bar{p} \bar{q}, p q}^{m}\left(d_{T}, d_{R}, \omega\right) \\
& +S_{\bar{p} \bar{q}, p q}^{\operatorname{LoS}}\left(d_{T}, d_{R}, \omega\right) .
\end{aligned}
$$

The SD-PSD of the LoS component can be written as

$$
\begin{aligned}
& S_{\bar{p} \bar{q}, p q}^{\mathrm{LoS}}\left(d_{T}, d_{R}, \omega\right) \\
& \quad=2 \pi \rho_{p q}^{2} \exp \left(j 2 \pi \Delta f_{\rho}\right) \delta\left(\omega-2 \pi f_{p q, \rho}\right),
\end{aligned}
$$

where $\delta(\bullet)$ is the Dirac delta function.

Since the ST-CF of the $m \mathrm{~B}$ component is the multiple integral as (28), the closed-form expression of SD-PSD cannot be derived. In Section 4.2, we show the SD-PSD of the $3 \mathrm{D} V 2 \mathrm{~V}$ channel in the presence of multiple moving scatterers by means of numerical integrations.

\section{Numerical Results and Validation}

This section demonstrates the normalized correlation functions and validates the Doppler power spectral density (DPSD) described in Section 3 through the MATLAB numerical simulations. Unless indicated otherwise, the values of the numerical simulation parameters are summarized in Table 2.

4.1. Numerical Results. In this section, the numerical curves of ST-CFs, T-CFs, and S-CFs influenced by some important contributory factors are presented. As in the typical urban environments, the power weight of the $m$ th cluster of rays has been set to $p_{1}=p_{2}=1 / 2$ for $M=2, p_{1}=p_{2}=p_{3}=1 / 3$ for $M=3, p_{1}=p_{2}=1 / 3, p_{3}=p_{4}=1 / 6$ for $M=4, p_{1}=1 / 3$, $p_{2}=1 / 4, p_{3}=p_{4}=1 / 6$, and $p_{5}=1 / 12$ for $M=5$.

4.1.1. ST-CFs and T-CFs for Different Scattering Scenarios and Bounces. Figures 2-5 demonstrate the ST-CFs and T-CFs for the different maximum bounces $M$ in the $3 \mathrm{D}$ isotropic and

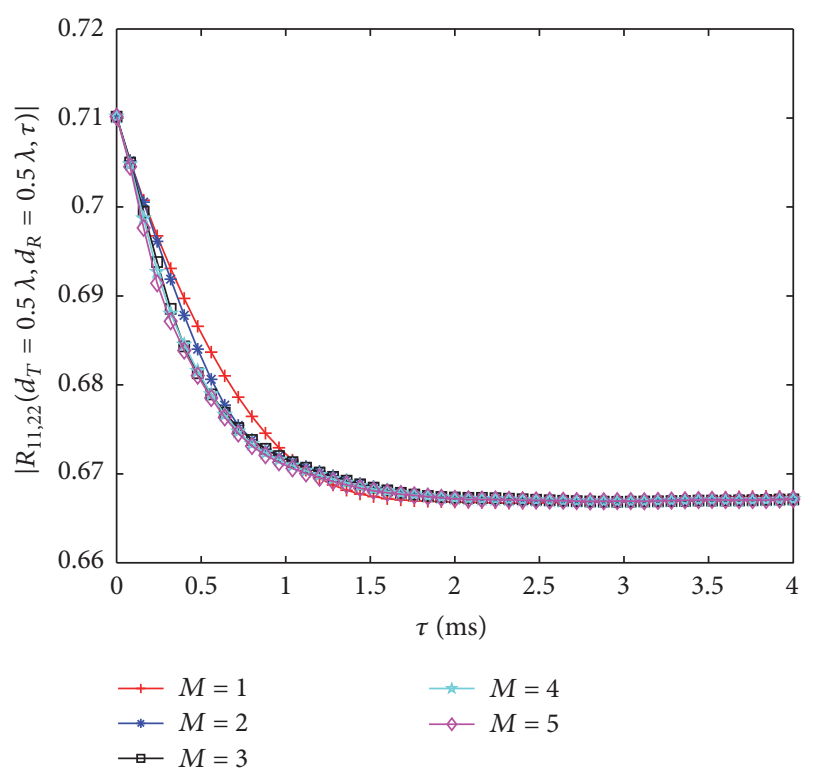

FIGURE 2: ST-CFs in (16) in the isotropic scattering scenario for different maximum bounces.

nonisotropic scattering scenarios. The scatterer velocity is uniformly distributed with an average speed of $25 \mathrm{~m} / \mathrm{s}$ that may be the velocity of passing vehicles. The other parameters used to obtain curves in Figures 2-5 are summarized in (Table 2, Cols. 2 and 3). As shown in Figures 2 and 4, the larger $M$ is, the faster the ST-CFs and T-CFs decrease in the isotropic scattering scenario. However, the descent rates of ST-CFs and T-CFs increase slowly when $M$ is larger than 3 . The similar conclusions can be obtained from Figures 3 and 5; however, the ST-CF curves in Figure 3 have oscillations which may be caused by the nonisotropic scattering. However, this conclusion differs from [27] which showed that the tripleor higher-order bounced rays had statistical properties very similar to those of the double-bounced rays and could be approximated as double-bounced rays. The discrepancy may be caused by the different communication environments considered by us and [27]. Specifically, channel-sounding experimental campaign in [27] was conducted along surface 


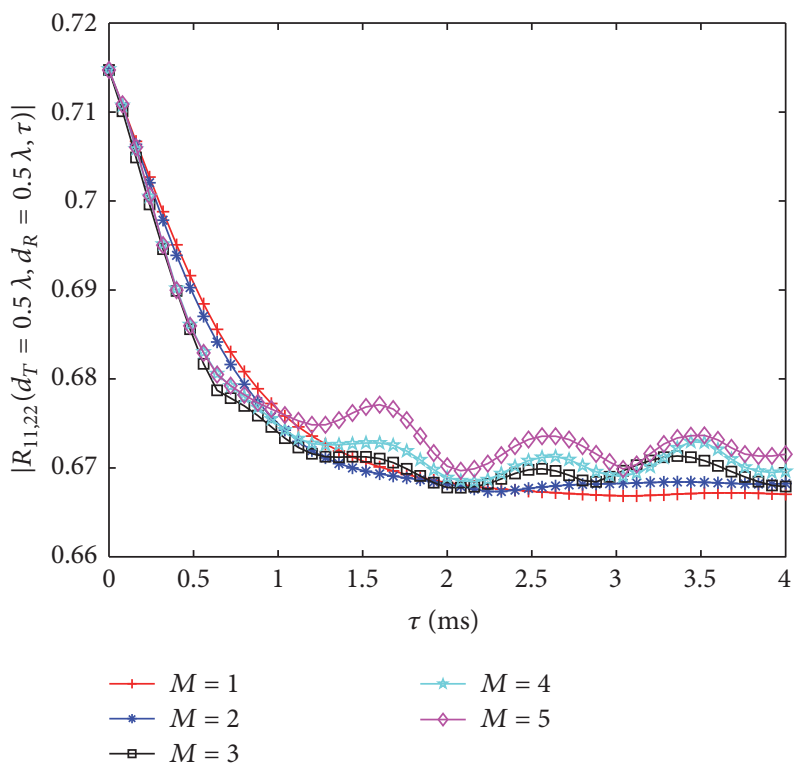

FIGURE 3: ST-CFs in (16) in the nonisotropic scattering scenario for different maximum bounces.

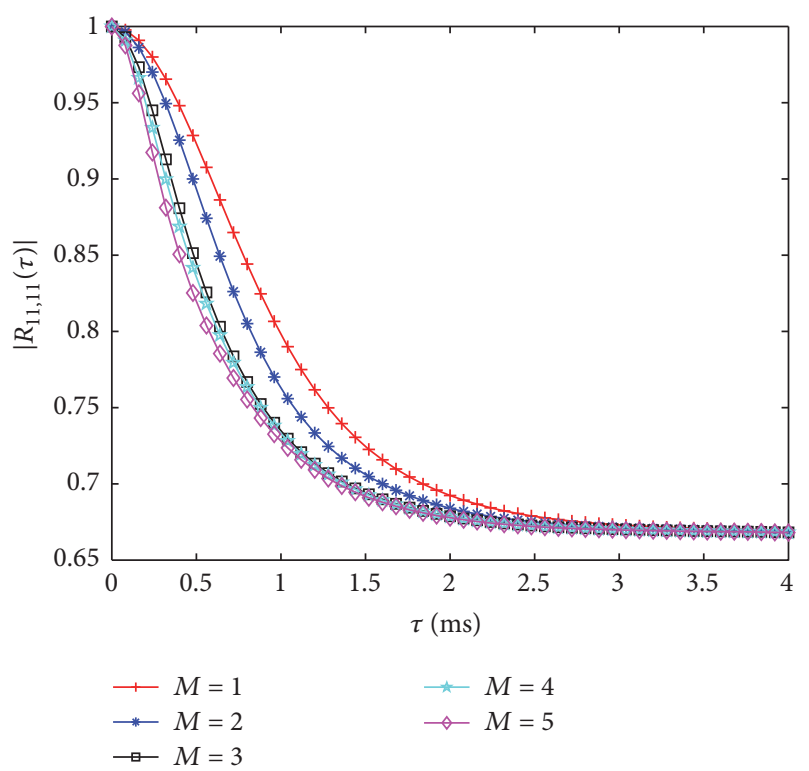

FIgURE 4: T-CFs in (16) with $p=\bar{p}$ and $q=\bar{q}$ in the isotropic scattering scenario for different maximum bounces.

streets around the Georgia Tech campus and on the Interstate highways in the Midtown Atlanta metropolitan area which may have lower dense scatterers than our communication environments. The ST-CF curves in Figure 3 converge relatively slower for the larger $M$ that means there are more local scatterers in motion with random velocities and random directions. As can be observed in Figures 4 and 5, the T-CFs in both figures are very similar that indicates that the scattering forms have no significant effect on the T-CFs of the 3D V2V channel in the presence of multiple moving scatterers. Furthermore, in Figures 2-5, it can be observed that a tendency to a limit value curve of ST-CFs and T-CFs can be inferred if

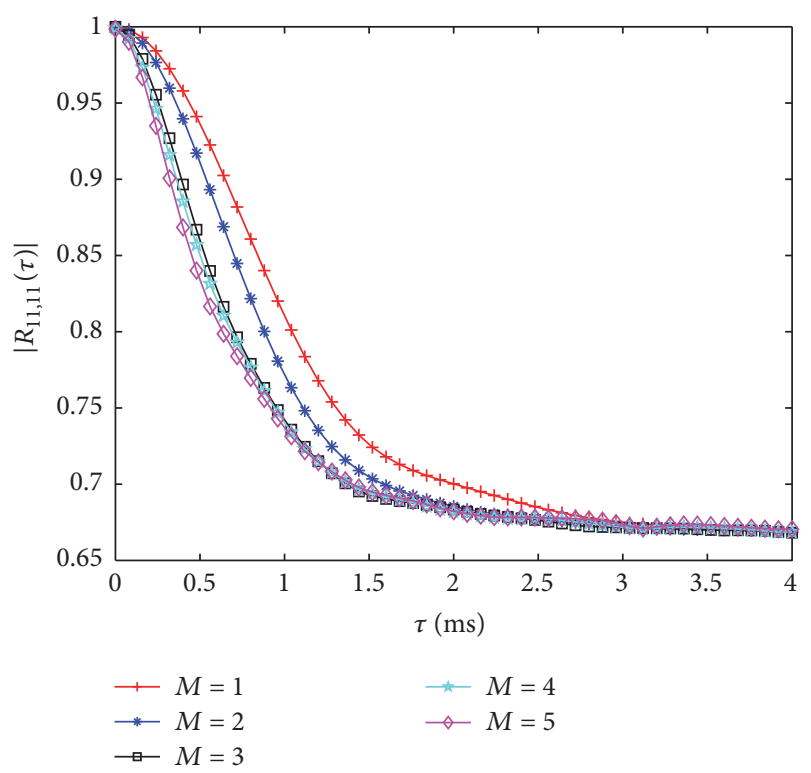

FIGURE 5: T-CFs in (16) with $p=\bar{p}$ and $q=\bar{q}$ in the nonisotropic scattering scenario for different maximum bounces.

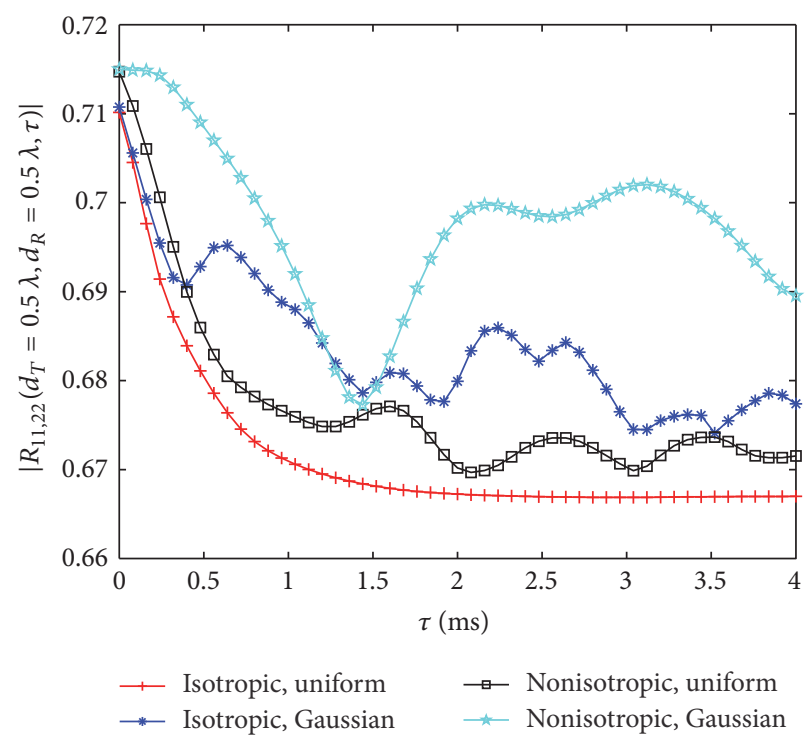

FIGURE 6: ST-CFs in (16) in the isotropic and nonisotropic scattering scenarios with $M=5$ for different scatterer velocity distributions.

$M$ approaches infinity, and the similar inference also can be obtained in (35) for the 2D scattering environment.

\subsubsection{ST-CFs and T-CFs for Different Scattering Scenarios and} Scatterer Velocity Distributions. Figures 6 and 7 demonstrate the ST-CFs and T-CFs for the different scatterer velocity distributions in the 3D isotropic and nonisotropic scattering scenarios. We use two uniform distributions in the isotropic scattering environment and use the von Mises distribution and the cosine distribution in the nonisotropic scattering environment to describe all the statistical azimuth and elevation angles in Table 1, respectively. The average speed of the uniform and half-Gaussian distributions is $25 \mathrm{~m} / \mathrm{s}$, 


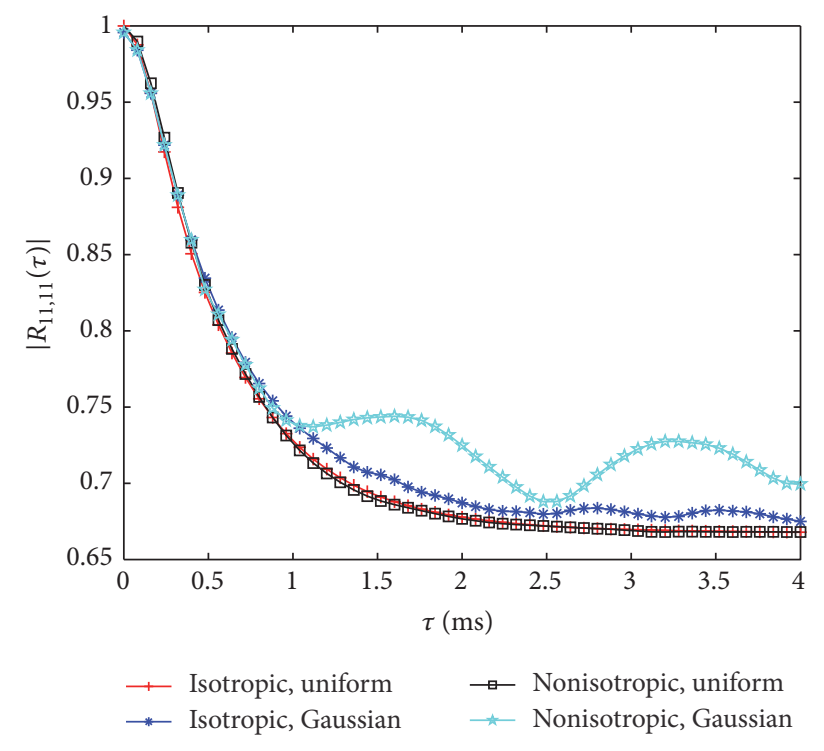

Figure 7: T-CFs in (16) with $p=\bar{p}$ and $q=\bar{q}$ in the isotropic and nonisotropic scattering scenarios with $M=5$ for different scatterer velocity distributions.

and the standard deviation of the half-Gaussian distribution is $31 \mathrm{~m} / \mathrm{s}$. The other parameters used to obtain curves in Figures 6 and 7 are summarized in (Table 2, Cols. 2 and 3). As shown in Figure 6, the ST-CF for the uniform distributed velocity of scatterers in the isotropic scattering environment decreases fastest, and both the nonisotropic scattering and the half-Gaussian distribution can result in swift oscillations of the fading curves. The comparison results indicate that the scatterer velocity distributions have more significant effect than the scattering forms on the ST-CFs of the $3 \mathrm{D} V 2 \mathrm{~V}$ channel in the presence of multiple moving scatterers. Figure 7 indicates that, not like scattering forms, the scatterer velocity distributions have significant effect on the T-CFs. Generally, the realistic propagation environment is nonisotropic scattering, and the scatterer velocity can be modeled by the half-Gaussian or uniform distribution. Therefore, the results of Figures 6 and 7 may be helpful in analysis of the system performance and modeling a specific propagation channel for $\mathrm{V} 2 \mathrm{~V}$ communications.

\subsubsection{ST-CFs and S-CFs for Different Scattering Scenarios} and Spacing between Adjacent Antenna Elements. Figures 8 and 9 demonstrate the 3D ST-CFs and S-CFs for the different spacing between adjacent antenna elements of $T_{X}$ and $R_{X}$ in isotropic and nonisotropic scattering scenarios. The set of the scatterer velocity in Figure 8 is the same as Figures 2-5, and the other parameters used to obtain curves in Figures 8 and 9 are summarized in (Table 2, Col. 4). Figures 8 and 9 show that spacing between adjacent antenna elements has significant effect on the 3D ST-CFs and S-CFs. Furthermore, the 3D ST-CFs in isotropic and nonisotropic scattering scenarios decrease to the bottom when $d_{R}=d_{T}$ tends to $1.2 \lambda$ and $0.7 \lambda$, respectively. In addition, the $3 \mathrm{D}$ S-CF in nonisotropic scattering scenarios is explicitly less than that in isotropic scattering scenarios for

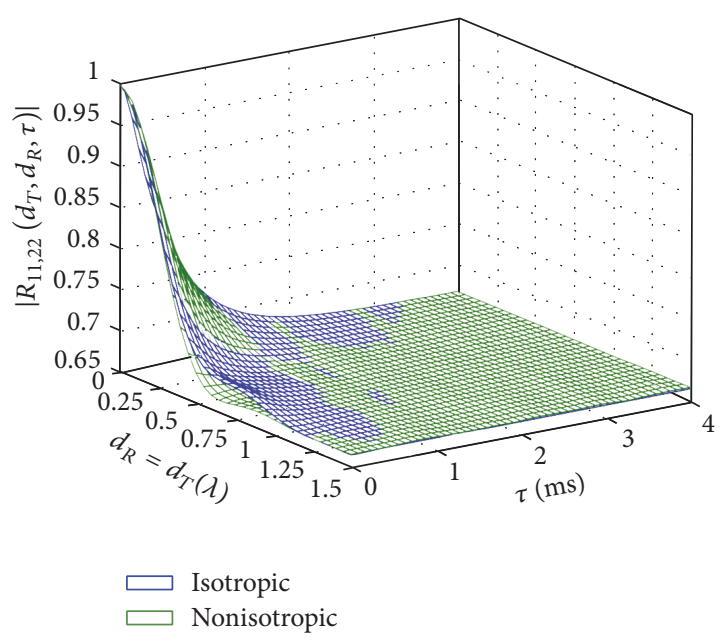

FIGURE 8: 3D ST-CFs in (16) in the isotropic and nonisotropic scattering scenarios with $M=5 . \lambda$ is the carrier wavelength.

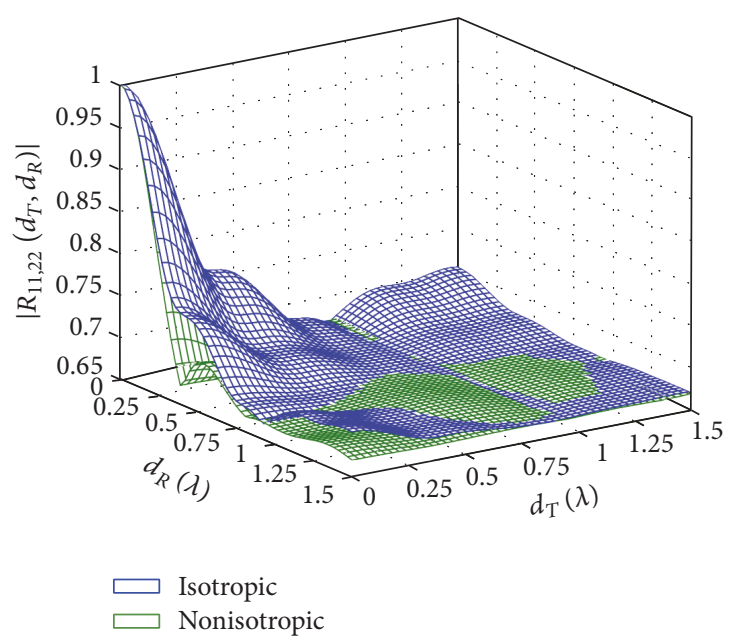

FIGURE 9: 3D S-CFs in (16) with $\tau=0$ in the isotropic and nonisotropic scattering scenarios. $\lambda$ is the carrier wavelength.

most spacing between adjacent antenna elements as shown in Figure 9. Therefore, the large-scale antenna arrays employing the spatial multiplexing are more suitable to be used in the nonisotropic scattering scenarios owing to the high antenna space utilization. The spacing between adjacent antenna elements of antenna arrays is suggested to be more than $\lambda$ in the $\mathrm{V} 2 \mathrm{~V}$ communications, especially in the environments with low-density scatterers.

4.2. Comparison with Measurements. The parametric nature of the proposed channel model makes it adaptable to a variety of propagation environments. D-PSD is one of the most important and unique channel characteristics for $\mathrm{V} 2 \mathrm{~V}$ communication channels. To illustrate the validity of the proposed model, we compare the modeled SD-PSDs of the $3 \mathrm{D}$ V2V channel with multiple moving scatterers with the measured F2F D-PSD in [26], the theoretical V2V SD-PSD in [18], and the measured V2V SD-PSD in [27]. 


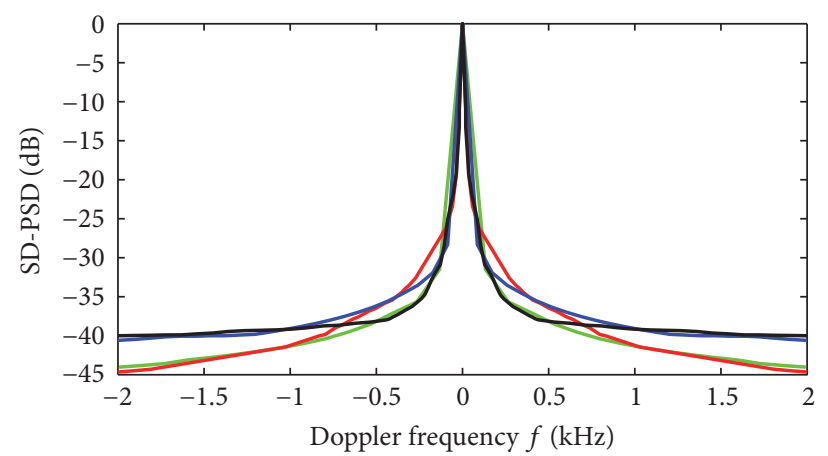

$\begin{array}{lll}\text { Modeled SD-PSD (foliage) } & - \text { Modeled SD-PSD (vehicles) } \\ \text { Measured D-PSD (foliage) } & - \text { Measured D-PSD (vehicles) }\end{array}$

FIGURE 10: Comparison between the modeled SD-PSDs with $M=5$ and the measured D-PSDs of Figure 4 in [26].

Figure 10 shows the comparison between the modeled SD-PSDs obtained from the Fourier transform of the STCF presented in (16) in the isotropic scattering scenario and the measured D-PSDs of Figure 4 in [26]. The outdoor measurement experiments in [26] have shown that, at millimeter wavelengths, fading caused by foliage movement and the motion of nearby vehicles is a significantly deleterious effect in systems with static subscribers. Figure 4 in [26] shows the D-PSD generated by foliage movements and the D-PSD caused by passing vehicles, both in a fixed wireless channel at a carrier frequency of $29.5 \mathrm{GHz}$. We have used the halfGaussian distribution with an average speed of $0.4 \mathrm{~m} / \mathrm{s}$ and the standard deviation of $0.5 \mathrm{~m} / \mathrm{s}$ to model the velocity of moving foliage scatterers, while the uniform distribution with an average speed of $6 \mathrm{~m} / \mathrm{s}$ has been used to model the velocity of passing vehicles scatterers. The power weight of the $m$ th cluster of rays scattered by the moving foliage or passing vehicles has been set to $p_{1}=9 / 16, p_{2}=5 / 16, p_{3}=1 / 16$, and $p_{4}=p_{5}=1 / 32$. The other parameters used to obtain the modeled SD-PSD curves in Figure 10 are summarized in (Table 2, Col. 5). As can be observed in Figure 10, $40 \mathrm{~dB}$ fading bandwidths for the effect of the foliage and vehicles movements are $500 \mathrm{~Hz}$ and $1 \mathrm{kHz}$ approximately, respectively. Fade depth due to foliage is less than the variation due to passing vehicles. Close agreements between the modeled and measured results are shown in Figure 10. These close matches can be seen not only for relatively slow moving scatterers but also for relatively fast moving scatterers as well.

Figure 11 shows the comparison among the modeled SDPSD obtained from the Fourier transform of the ST-CF presented in (16) in the nonisotropic scattering scenario, the theoretical SD-PSD of Figure 8 in [18], and the measured SDPSD of Figure 11 in [27]. The theoretical SD-PSD reproduced here is based on the two-cylinder model with moving and stationary scatterers for $d_{R}=d_{T}=0$. The channel measurements in [27] for the measured SD-PSD were collected at $2.435 \mathrm{GHz}$ in the urban street surface environment, and the spacing between two adjacent antenna elements of $T_{X}$ and $R_{X}$ was set to zero. We have used the half-Gaussian distribution with average speeds of $2.394 \mathrm{~m} / \mathrm{s}, 0.798 \mathrm{~m} / \mathrm{s}$, and $0.4 \mathrm{~m} / \mathrm{s}$
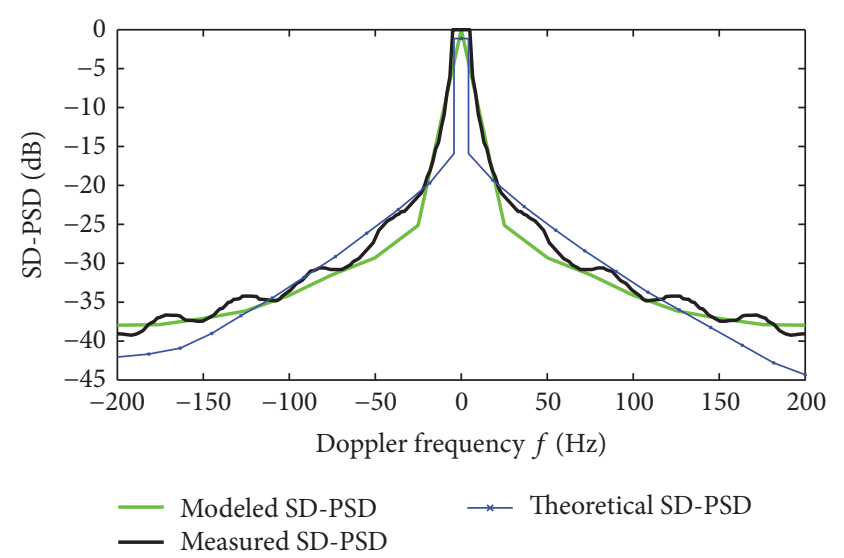

FIgURE 11: Comparison among the modeled SD-PSD with $M=5$, the theoretical SD-PSD of Figure 8 in [18], and the measured SDPSD of Figure 11 in [27].

and the standard deviation of $3 \mathrm{~m} / \mathrm{s}, 1 \mathrm{~m} / \mathrm{s}$, and $0.5 \mathrm{~m} / \mathrm{s}$ to model the velocity of the first, the second, and the remaining moving scatterers on the communication links, respectively. The power weight of the $m$ th cluster of rays has been set to $p_{1}=8 / 15, p_{2}=1 / 3, p_{3}=1 / 15, p_{4}=1 / 30$, and $p_{5}=1 / 30$ for $M=5$. The other parameters used to obtain the modeled SD-PSD curve in Figure 11 are summarized in (Table 2, Col. $6)$. We note that, different from the measured SD-PSDs in [26], the measured SD-PSD curve in [27] has oscillations. The oscillating pattern in measurements for the case $d_{R}=d_{T}=0$ may appear because it is not a true SISO scenario where only one transmit and one receive antenna are active [27]. As can be observed in Figure 11, the modeled SD-PSD matches better with measured SD-PSD than the theoretical SD-PSD in [18]. This may be because of the introduction of the multiple bounced rays into the propagation model or the constraints on the position of local scatterers in the two-cylinder model proposed in [18].

Figure 12 demonstrates the CDFs of the relative deviations between modeled/theoretical SD-PSDs and corresponding measured values in Figures 10 and 11. The mean relative deviation of the modeled SD-PSDs for foliage in Figure 10, modeled SD-PSDs for vehicles in Figure 10, modeled SD-PSDs in Figure 11, and theoretical SD-PSDs in Figure 11 is $2.56 \%, 2.16 \%, 3.9 \%$, and $8.32 \%$, respectively. As shown in Figure 12, more than $80 \%$ of the relative deviations of modeled SD-PSDs in Figures 10 and 11 are less than $5 \%$, and the relative deviations of theoretical SD-PSDs in Figure 11 are explicitly larger than those of modeled SDPSDs. In addition, from Figures 10 and 11, we note that the modeled SD-PSD matches better with measured SD-PSDs at the lower and higher Doppler frequencies than the middle Doppler frequencies. This may be because $T_{X}$ and $R_{X}$ were not strictly fixed, and not all the scatterers were in motion in measurements. The approximate calculation of the frequency shift in (A.4) and Doppler shift in (10) may be another factor which causes the mismatch at the middle Doppler frequencies, and more investigations concerning the accuracy of the proposed model will be addressed in future works. 


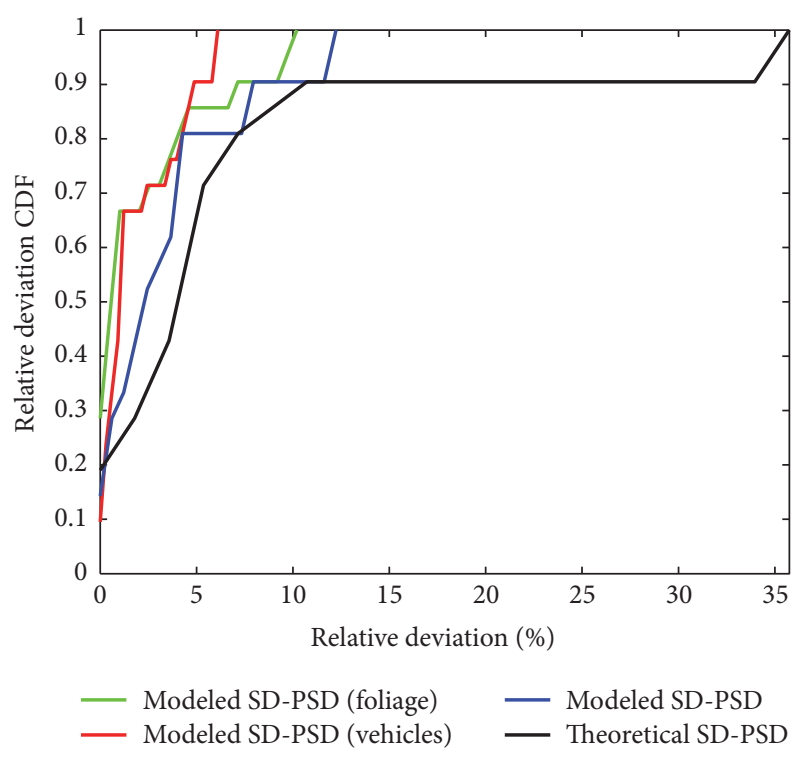

FIGURE 12: The relative deviation cumulative distribution functions (CDFs) of the modeled and theoretical SD-PSDs in Figures 10 and 11.

However, as shown in Figures 10-12, the acceptable matches confirm the utility and generality of the proposed model and show the need for including multiple moving scatterers in propagation model.

\section{Conclusion}

Without specific constraints on the position of the local moving scatterers, a 3D geometrical V2V propagation model that includes LoS, single bounced, and multiple bounced links between the transmitter and receiver was proposed. Based on the geometrical propagation model, a 3D reference model for narrowband MIMO V2V multipath fading channels was developed. From the reference model, the corresponding mathematical expressions and numerical results of ST-CFs, T-CFs, S-CFs, and SD-PSDs were studied for different parametric sets. It has been shown that the maximum bounces, scattering forms, scatterer velocity distributions, and spacing between adjacent antenna elements have significant effect on the ST-CFs, and only the maximum bounces and scatterer velocity distributions have significant effect on the T-CFs. Finally, the modeled SD-PSD results were compared with the measured data and the other literature's theoretical result. The close agreements between the analytically and empirically obtained channel statistics confirmed the utility and generality of the proposed model.

\section{Appendix}

\section{A. Derivation Process of Frequency Shifts}

The derivation process of $f_{p q, n}^{q}$ in (7) is presented in this section, and $f_{p q, \rho}^{p}, f_{p q, n}^{q}, f_{p q, n}^{p}$, and $f_{p q, \rho}$ can be calculated similarly.

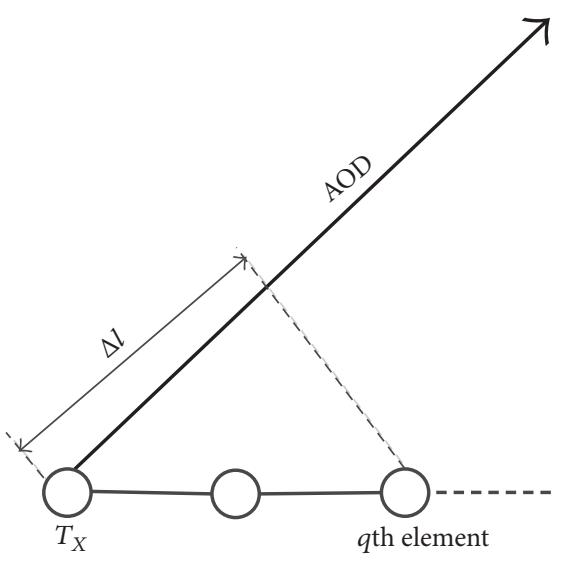

FIGURE 13: Projection from the coordinate vector of $q$ th antenna element to the AOD direction.

According to Figure 1, the coordinate vectors of $q$ th antenna element and the AOD direction can be written as, respectively,

$$
\begin{aligned}
& \vec{L}_{q} \\
& \quad=(q-1) d_{T}\left(\cos \beta_{q} \cos \alpha_{q}, \cos \beta_{q} \sin \alpha_{q}, \sin \beta_{q}\right), \\
& \vec{L}_{\mathrm{AOD}}=\left(\cos \beta_{p q}^{T} \cos \alpha_{p q}^{T}, \cos \beta_{p q}^{T} \sin \alpha_{p q}^{T}, \sin \beta_{p q}^{T}\right) .
\end{aligned}
$$

Therefore, the projection $\Delta l$ in Figure 13 is

$$
\begin{aligned}
\Delta l & =\frac{\vec{L}_{q} \cdot \vec{L}_{\mathrm{AOD}}}{\left|\vec{L}_{\mathrm{AOD}}\right|}=(q \\
& -1) d_{T}\left[\cos \left(\alpha_{q}-\alpha_{p q}^{T}\right) \cos \beta_{q} \cos \beta_{p q}^{T}+\sin \beta_{q} \sin \beta_{p q}^{T}\right] .
\end{aligned}
$$

Because we mainly consider the difference of the frequency shifts caused by the TPD changes to calculate ST-CFs, the TPD change of $h_{11}(t)$ is assumed to be zero. Eventually, $f_{p q, n}^{q}$ can be calculated as

$$
\begin{aligned}
f_{p q, n}^{q} & \approx \frac{\Delta l f_{0}}{c} \\
& =\frac{(q-1) d_{T} f_{0}}{c}\left[\cos \left(\alpha_{q}-\alpha_{p q}^{T}\right) \cos \beta_{q} \cos \beta_{p q}^{T}\right. \\
& \left.+\sin \beta_{q} \sin \beta_{p q}^{T}\right] .
\end{aligned}
$$

\section{B. Derivation Process of Approximate ST-CFs}

In the $2 \mathrm{D}$ scattering environment, the simplified and approximate expressions of (28) can be derived in this section. If $\beta_{T}=\beta_{R}=\beta_{p q}^{T}=\beta_{p q}^{R}=\beta_{p q}^{i}=0,(27)$ and (10) can be written as

$$
\begin{gathered}
\Delta f_{n_{m}}=\frac{f_{0}}{c}\left[(q-\bar{q}) d_{T} \cos \left(\alpha_{q}-\alpha_{p q}^{T}\right)\right. \\
\left.+(p-\bar{p}) d_{R} \cos \left(\alpha_{p}-\alpha_{p q}^{R}\right)\right],
\end{gathered}
$$




$$
\begin{gathered}
f_{p q, n_{m}}=\frac{f_{0}}{c}\left[v_{T} \cos \left(\alpha_{T}-\alpha_{p q}^{T}\right)+v_{R} \cos \left(\alpha_{R}-\alpha_{p q}^{R}\right)\right. \\
+v_{p q}^{1} \cos \left(\alpha_{p q}^{1}-\alpha_{p q}^{T}\right)+2 \sum_{i=2}^{m-1} v_{p q}^{i} \cos \left(\alpha_{p q}^{i}-\alpha_{p q}^{T}\right) \\
+v_{p q}^{m} \cos \left(\alpha_{p q}^{m}-\alpha_{p q}^{T}\right)+v_{p q}^{1} \cos \left(\alpha_{p q}^{1}-\alpha_{p q}^{R}\right) \\
\left.+2 \sum_{i=2}^{m-1} v_{p q}^{i} \cos \left(\alpha_{p q}^{i}-\alpha_{p q}^{R}\right)+v_{p q}^{m} \cos \left(\alpha_{p q}^{m}-\alpha_{p q}^{R}\right)\right] .
\end{gathered}
$$

If $\alpha_{T}=\alpha_{q}$ and $\alpha_{R}=\alpha_{p}$, that is, the antenna array and its velocity have the same directions, (28) can be simplified as by substituting (B.1) into (28)

$$
\begin{aligned}
& R_{\overline{p q}, p q}^{m}\left(d_{T}, d_{R}, \tau\right) \\
& =2 \sigma_{m}^{2} \int \cdots \iint \cdots \iiint \exp \left\{j k _ { 0 } \left[\left[v_{T} \tau+(q-\bar{q}) d_{T}\right]\right.\right. \\
& \times \cos \left(\alpha_{T}-\alpha_{p q}^{T}\right)+\left[v_{R} \tau+(p-\bar{p}) d_{R}\right] \cos \left(\alpha_{R}-\alpha_{p q}^{R}\right) \\
& +2 v_{p q}^{1} \tau \cos \left(\frac{\alpha_{p q}^{T}-\alpha_{p q}^{R}}{2}\right) \cos \left(\alpha_{p q}^{1}-\frac{\alpha_{p q}^{T}+\alpha_{p q}^{R}}{2}\right) \\
& +4 \tau \cos \left(\frac{\alpha_{p q}^{T}-\alpha_{p q}^{R}}{2}\right) \sum_{i=2}^{m-1} v_{p q}^{i} \cos \left(\alpha_{p q}^{i}-\frac{\alpha_{p q}^{T}+\alpha_{p q}^{R}}{2}\right) \\
& \left.\left.+2 v_{p q}^{m} \tau \cos \left(\frac{\alpha_{p q}^{T}-\alpha_{p q}^{R}}{2}\right) \cos \left(\alpha_{p q}^{m}-\frac{\alpha_{p q}^{T}+\alpha_{p q}^{R}}{2}\right)\right]\right\} \prod_{i=1}^{m} p\left(v_{p q}^{i}\right) \\
& +\prod_{i=1}^{m} p\left(\alpha_{p q}^{i}\right) p\left(\alpha_{p q}^{T}\right) p\left(\alpha_{p q}^{R}\right) d v_{p q}^{1} \cdots d v_{p q}^{m} d \alpha_{p q}^{1} \cdots d \alpha_{p q}^{m} d \alpha_{p q}^{T} d \alpha_{p q}^{R},
\end{aligned}
$$

where $k_{0}=2 \pi f_{0} / c$ is the wave number.

In isotropic scattering environment, we use the uniform distribution in (31) to describe all the random azimuth angles. In this case, (B.2) can be written as

$$
\begin{aligned}
& R_{\overline{p q}, p q}^{m}\left(d_{T}, d_{R}, \tau\right) \\
& =\frac{\sigma_{m}^{2}}{2 \pi^{2}} \int \cdots \iiint \exp \left\{j k_{0}\left[v_{T} \tau+(q-\bar{q}) d_{T}\right] \cos \left(\alpha_{T}-\alpha_{p q}^{T}\right)\right. \\
& \left.+j k_{0}\left[v_{R} \tau+(p-\bar{p}) d_{R}\right] \cos \left(\alpha_{R}-\alpha_{p q}^{R}\right)\right\} \\
& \times J_{0}\left[2 k_{0} v_{p q}^{1} \tau \cos \left(\frac{\alpha_{p q}^{T}-\alpha_{p q}^{R}}{2}\right)\right] \\
& \times J_{0}\left[2 k_{0} v_{p q}^{m} \tau \cos \left(\frac{\alpha_{p q}^{T}-\alpha_{p q}^{R}}{2}\right)\right] \\
& \times \prod_{i=2}^{m-1} J_{0}\left[4 k_{0} v_{p q}^{i} \tau \cos \left(\frac{\alpha_{p q}^{T}-\alpha_{p q}^{R}}{2}\right)\right] \\
& . \prod_{i=1}^{m} p\left(v_{p q}^{i}\right) d v_{p q}^{1} \cdots d v_{p q}^{m} d \alpha_{p q}^{T} d \alpha_{p q}^{R}
\end{aligned}
$$

where $J_{0}(\bullet)$ denotes the zeroth-order Bessel function of the first kind.

The AOD $\alpha_{p q}^{T}$ and AOA $\alpha_{p q}^{R}$ are uniformly and independently distributed, so $\alpha_{p q}^{T}-\alpha_{p q}^{R}$ can be equal to zero in average.
As a consequence, the term $\cos \left[\left(\alpha_{p q}^{T}-\alpha_{p q}^{R}\right) / 2\right]$ in (B.3) can be approximated by one $[13,14]$, and an approximate ST-CF can be written as from (B.3)

$$
\begin{aligned}
& R_{\bar{p} \bar{q}, p q}^{m}\left(d_{T}, d_{R}, \tau\right) \\
& =\frac{\sigma_{m}^{2}}{2 \pi^{2}} \int \cdots \iiint \exp \left\{j k_{0}\left[v_{T} \tau+(q-\bar{q}) d_{T}\right]\right. \\
& \cdot \cos \left(\alpha_{T}-\alpha_{p q}^{T}\right) \\
& +j k_{0}\left[v_{R} \tau+(p-\bar{p}) d_{R}\right] \\
& \left.\cdot \cos \left(\alpha_{R}-\alpha_{p q}^{R}\right)\right\} J_{0}\left(2 k_{0} v_{p q}^{1} \tau\right) \\
& \times J_{0}\left(2 k_{0} \nu_{p q}^{m} \tau\right) \prod_{i=2}^{m-1} J_{0}\left(4 k_{0} \nu_{p q}^{i} \tau\right) \\
& \cdot \prod_{i=1}^{m} p\left(v_{p q}^{i}\right) d v_{p q}^{1} \cdots d v_{p q}^{m} d \alpha_{p q}^{T} d \alpha_{p q}^{R} .
\end{aligned}
$$

Eventually, the approximate ST-CF in (34) can be derived from (B.4) according to the definition of $J_{0}(\bullet)$.

\section{Conflicts of Interest}

The authors declare that they have no conflicts of interest.

\section{Acknowledgments}

This work was funded by the National Natural Science Foundation of China (no. 91438104, no. 61501065, no. 61571069, and no. 61601067), the Fundamental Research Funds for the Central Universities (no. 106112016CDJX160001), and the Chongqing Research Program of Basic Research and Frontier Technology (no. CSTC2016JCYJA0021).

\section{References}

[1] A. Bazzi, B. M. Masini, A. Zanella, and I. Thibault, "Beaconing from connected vehicles: IEEE 802.11p vs. LTE-V2V," in Proceedings of 2016 IEEE 27th Annual International Symposium on Personal, Indoor, and Mobile Radio Communications (PIMRC), pp. 1-6, Valencia, Spain, September 2016.

[2] V. M. Rodrigo-Peñarrocha, J. Reig, L. Rubio, and et al., "Analysis of small- scale fading distributions in vehicle-to-vehicle communications," Mobile Information Systems, vol. 2016, Article ID 9584815, 7 pages, 2016.

[3] A. Bazzi, B. M. Masini, A. Zanella, and G. Pasolini, "IEEE 802.11p for cellular offloading in vehicular sensor networks," Computer Communications, vol. 60, pp. 97-108, 2015.

[4] X. Cheng, MIMO channel modelling and simulation for cellular and mobile-to-mobile communication systems[Phd thesis], Heriot-Watt University, 2009.

[5] N. Avazov and M. Pätzold, "A geometric street scattering channel model for car-to-car communication systems," in Proceedings of 4th Annual International Conference on Advanced 
Technologies for Communications, ATC2011, pp. 224-230, vnm, August 2011.

[6] X. Zhao, X. Liang, S. Li, and K. Haneda, "Mobile-to-Mobile Wideband MIMO Channel Realization by Using a Two-Ring Geometry-Based Stochastic Scattering Model," Wireless Personal Communications, vol. 84, no. 4, pp. 2445-2465, 2015.

[7] M. Walter, D. Shutin, and U.-C. Fiebig, "Delay-dependent doppler probability density functions for vehicle-to-vehicle scatter channels," IEEE Transactions on Antennas and Propagation, vol. 62, no. 4, pp. 2238-2249, 2014.

[8] P. T. Samarasinghe, T. A. Lamahewa, T. D. Abhayapala, and R. A. Kennedy, "3D mobile-to-mobile wireless channel model," in Proceedings of 2010 Australian Communications Theory Workshop, AusCTW 2010, pp. 30-34, aus, February 2010.

[9] M. Riaz, S. J. Nawaz, and N. M. Khan, "3D ellipsoidal model for mobile-to-mobile radio propagation environments," Wireless Personal Communications, vol. 72, no. 4, pp. 2465-2479, 2013.

[10] J. Chen and T. G. Pratt, "Three-dimensional geometrybased stochastic modeling and performance of $4 \times 4$ spacepolarization mobile-to-mobile wideband MIMO channels," in Proceedings of 2013 IEEE Global Communications Conference, GLOBECOM 2013, pp. 3936-3941, usa, December 2013.

[11] Y. Bi, J. Zhang, M. Zeng, M. Liu, and X. Xu, "A Novel 3D Nonstationary Channel Model Based on the von Mises-Fisher Scattering Distribution," Mobile Information Systems, vol. 2016, Article ID 2161460, 2016.

[12] A. Chelli and M. Patzöld, "The impact of fixed and moving scatterers on the statistics of MIMO vehicle-to-vehicle channels," in Proceedings of VTC Spring 2009 - IEEE 69th Vehicular Technology Conference, esp, April 2009.

[13] A. Borhani and M. Pätzold, "Modeling of vehicle-to-vehicle channels in the presence of moving scatterers," in Proceedings of 2012 IEEE Vehicular Technology Conference (VTC Fall), pp. 1-5, Quebec City, QC, Canada, September 2012.

[14] A. Borhani and M. Patzold, "Correlation and spectral properties of vehicle-to-vehicle channels in the presence of moving scatterers," IEEE Transactions on Vehicular Technology, vol. 62, no. 9, pp. 4228-4239, 2013.

[15] A. Chelli and M. Pätzold, "A non-stationary MIMO vehicleto-vehicle channel model derived from the geometrical street model," in Proceedings of the IEEE 74th Vehicular Technology Conference (VTC Fall '11), pp. 1-6, IEEE, San Francisco, Calif, USA, September 2011.

[16] M. D. Soltani, M. Alimadadi, Y. Seyedi, and H. Amindavar, "Modeling of doppler spectrum in V2V urban canyon oncoming environment," in Proceedings of 2014 7th International Symposium on Telecommunications, IST 2014, pp. 1155-1160, irn, September 2014.

[17] M. D. Soltani, M. Alimadadi, and A. Mohammadi, "Modeling of mobile scatterer clusters for doppler spectrum in wideband vehicle-to-vehicle communication channels," IEEE Communications Letters, vol. 18, no. 4, pp. 628-631, 2014.

[18] A. G. Zajić, "Impact of moving scatterers on vehicle-to-vehicle narrow-band channel characteristics," IEEE Transactions on Vehicular Technology, vol. 63, no. 7, pp. 3094-3106, 2014.

[19] A. G. Zajić, "Modeling impact of moving scatterers on doppler spectrum in wideband vehicle-to-vehicle channels," in Proceedings of Proc. Eur. Conf. Antennas Propag, p. 1, Lisbon, May 2015.

[20] J. Zhang, X. Yin, and X. Cheng, “Theoretical analysis and measurements: Doppler spectra of vehicular communication channels," in Proceedings of 2012 12th International Conference on ITS Telecommunications, ITST 2012, pp. 98-102, twn, November 2012.

[21] M. Picone, S. Busanelli, M. Amoretti, F. Zanichelli, and G. Ferrari, Advanced Technologies for Intelligent Transportation Systems, Springer, 2015.

[22] V. H. Pham, M. H. Taieb, J. Y. Chouinard, S. Roy, and H. T. Huynh, "On the double Doppler effect generated by scatterer motion," REV Journal on Electronics and Communications, vol. 1, pp. 30-37, 2011.

[23] S. Roy, H. T. Huynh, and P. Fortier, "Compound Doppler spread effects of subscriber motion and scatterer motion," $A E U$ - International Journal of Electronics and Communications, vol. 57, no. 4, pp. 237-246, 2003.

[24] J. B. Andersen, J. O. Nielsen, G. F. Pedersen, G. Bauch, and G. Dietl, "Doppler spectrum from moving scatterers in a random environment," IEEE Transactions on Wireless Communications, vol. 8, no. 6, pp. 3270-3277, 2009.

[25] H. S. Rad, S. Gazor, and P. Shariatpanahi, "Non-fixed scatterers and their effects on MIMO multicarrier fading communication channels," in Proceedings of 50th Annual IEEE Global Telecommunications Conference, GLOBECOM 2007, pp. 3765-3769, usa, November 2007.

[26] N. Naz and D. Falconer, "Temporal variations characterization for fixed wireless at $29.5 \mathrm{GHz}$," in Proceedings of 2000 IEEE 51st Vehicular Technology Conference. Proceedings. VTC2000Springer, pp. 2178-2182, Tokyo, Japan, 2000.

[27] A. G. Zajić, G. L. Stüber, T. G. Pratt, and S. T. Nguyen, "Wideband MIMO mobile-to-mobile channels: Geometrybased statistical modeling with experimental verification," IEEE Transactions on Vehicular Technology, vol. 58, no. 2, pp. 517-534, 2009.

[28] W. C. Jakes, Microwave Mobile Communications, Wiley-IEEE Press, Piscataway, NJ, USA, 1994.

[29] M. Pätzold, B. O. Hogstad, and N. Youssef, "Modeling, analysis, and simulation of MIMO mobile-to-mobile fading channels," IEEE Transactions on Wireless Communications, vol. 7, no. 2, pp. 510-520, 2008.

[30] M. Pätzold, Mobile Radio Channels, Wiley, Chichester, UK, 2nd edition, 2011.

[31] R. Wang and D. Cox, "Double mobility mitigates fading in ad hoc wireless networks," in Proceedings of IEEE Antennas and Propagation Society International Symposium, pp. 306-309, San Antonio, TX, USA.

[32] G. Bakhshi, K. Shahtalebi, and H. S. Rad, "A novel fullthree-dimensional MIMO mobile-to-mobile channel reference model," in Proceedings of 3rd International Conference on Signal Processing and Communication Systems, ICSPCS'2009, usa, September 2009.

[33] W. Zhou, X. Wang, X. Wang, and W. Chen, "A modified twoerose- ring model for mimo mobile-to-mobile fading channels," in Proceedings of 7th International Conference on Wireless Communications, Networking and Mobile Computing, WiCOM 2011, 5, 1 pages, Wuhan, China, September 2011. 

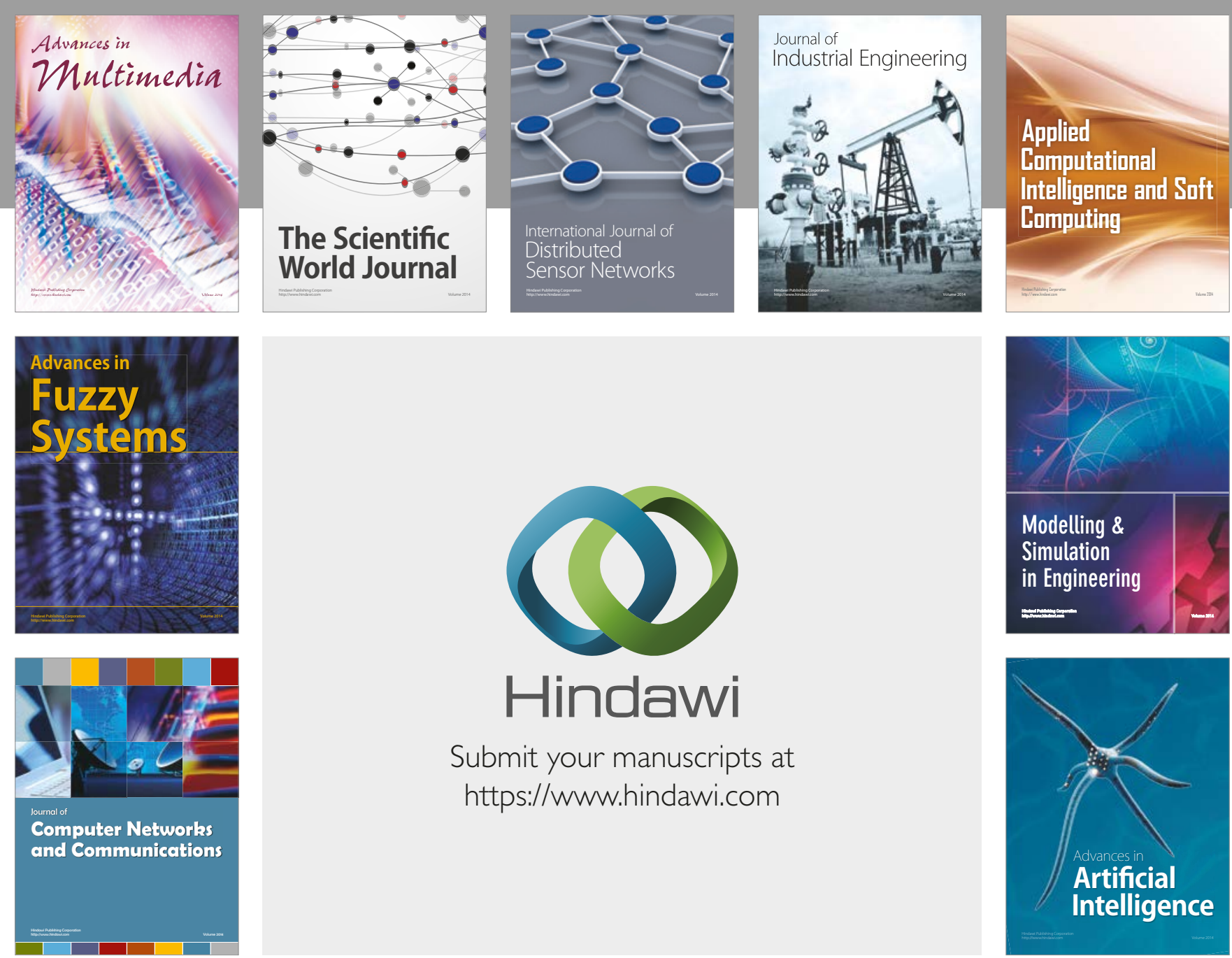

\section{Hindawi}

Submit your manuscripts at

https://www.hindawi.com
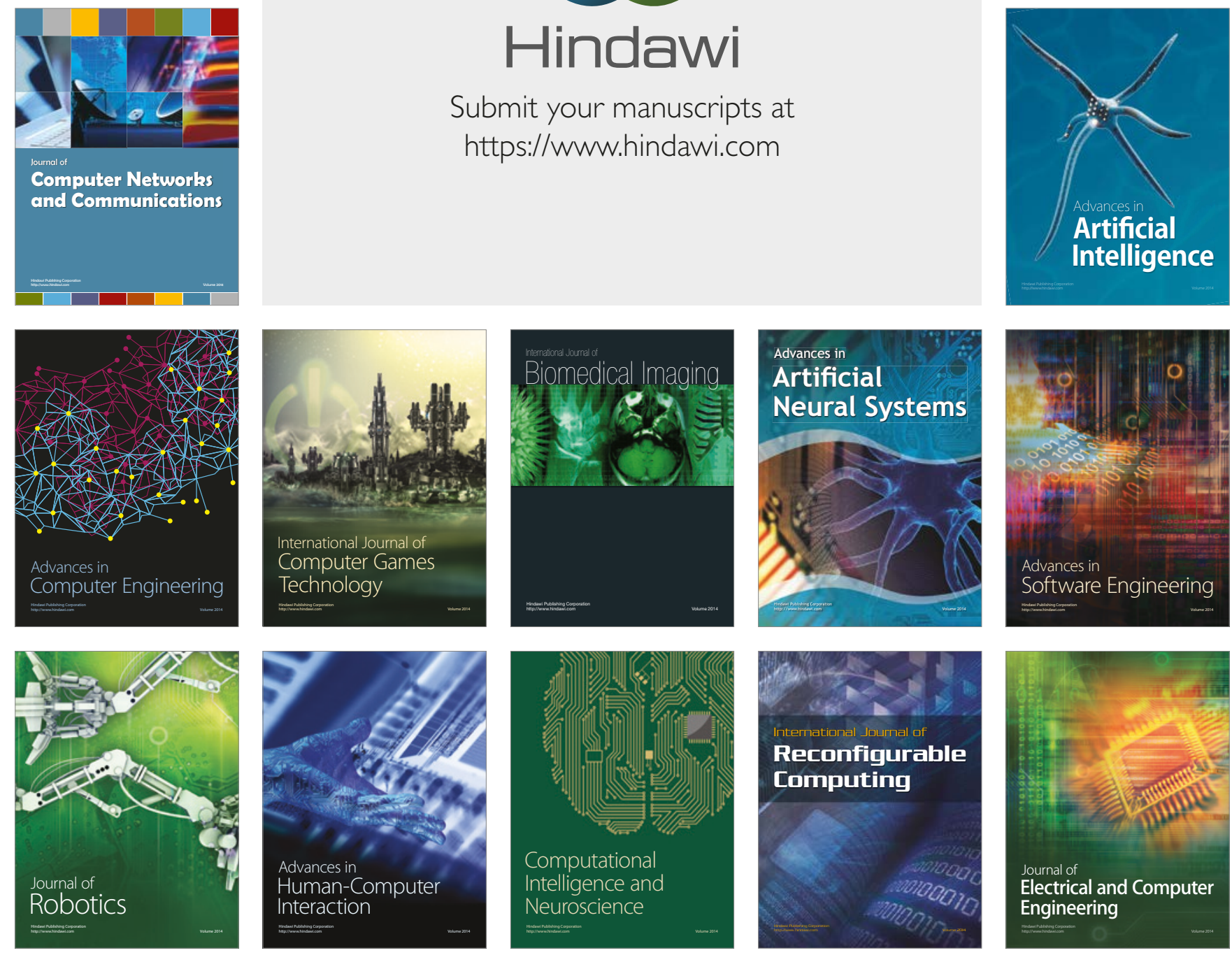